SNS-PH/00-16

UCB-PTH-00/38

LBNL-47128

\title{
A Constrained Standard Model from a Compact Extra Dimension
}

\author{
Riccardo Barbieri ${ }^{a}$, Lawrence J. Hall ${ }^{b, c}$, Yasunori Nomura ${ }^{b, c}$ \\ a Scuola Normale Superiore and INFN, Piazza dei Cavalieri 7, I-56126 Pisa, Italy \\ ${ }^{b}$ Department of Physics, University of California, Berkeley, CA 94720, USA \\ c Theoretical Physics Group, Lawrence Berkeley National Laboratory, Berkeley, CA 94720, USA
}

\begin{abstract}
A $S U(3) \times S U(2) \times U(1)$ supersymmetric theory is constructed with a TeV sized extra dimension compactified on the orbifold $S^{1} /\left(Z_{2} \times Z_{2}^{\prime}\right)$. The compactification breaks supersymmetry leaving a set of zero modes which correspond precisely to the states of the 1 Higgs doublet standard model. Supersymmetric Yukawa interactions are localized at orbifold fixed points. The top quark hypermultiplet radiatively triggers electroweak symmetry breaking, yielding a Higgs potential which is finite and exponentially insensitive to physics above the compactification scale. This potential depends on only a single free parameter, the compactification scale, yielding a Higgs mass prediction of $127 \pm 8 \mathrm{GeV}$. The masses of the all superpartners, and the Kaluza-Klein excitations are also predicted. The lightest supersymmetric particle is a top squark of mass $197 \pm 20 \mathrm{GeV}$. The top Kaluza-Klein tower leads to the $\rho$ parameter having quadratic sensitivity to unknown physics in the ultraviolet.
\end{abstract}




\section{Introduction}

The standard model provides an economical description of particles and their interactions in terms of 18 free parameters. There are 9 parameters associated with the masses of the quarks and charged leptons, and 4 to describe the flavor mixing of the quarks. There are 3 independent gauge couplings, and the final 2 parameters are associated with the Higgs boson. One is the vacuum expectation value (VEV) of the Higgs field, which is accurately determined by the Fermi coupling constant, and the other is the mass of the Higgs boson, which is unknown, although precision electroweak data suggests it is less than $188 \mathrm{GeV}$ at $95 \%$ confidence level [1].

Despite the phenomenological success and mathematical consistency of the standard model, it does not provide a physical description of electroweak symmetry breaking (EWSB). The theory is believed to be an effective theory valid at all energies below some cutoff, $\Lambda$. Yet the mass parameter of the Higgs field has radiative corrections that grow quadratically with $\Lambda$

$$
m_{H}^{2} \propto-\Lambda^{2}
$$

The physics of EWSB is at or beyond the cutoff, and hence not adequately described by the low energy effective theory.

In this paper we introduce a theory that does provide a full physical description of EWSB, in terms of new physics at a mass scale of $400 \mathrm{GeV}$. Our theory contains 17 free parameters, one fewer than the standard model, so that we are able to predict the mass of the Higgs boson.

A key feature of most theories which go beyond the standard model is an enhanced symmetry structure. Symmetries are the key to constructing more predictive and elegant theories. Yet they are also a challenge, because nature does not possess these additional symmetries, so they must be broken and this often introduces great freedom. For example, grand unified theories provide

a relation between the three gauge coupling constants, and yet breaking the grand unified gauge symmetry is the least attractive and constrained aspect of these theories.

It is commonly believed that the most satisfactory way to construct a physical theory of EWSB is to extend spacetime symmetry to include supersymmetry [2]. In this case, the quadratic divergence in the Higgs boson mass parameter coming from a top quark radiative correction is cancelled by that coming from a scalar top. Including supersymmetry breaking at scale $m_{\mathrm{SUSY}}$, the resulting divergence is logarithmic

$$
m_{H}^{2} \propto-m_{\mathrm{SUSY}}^{2} \ln \Lambda
$$

so that EWSB may be triggered by physics all the way up to the cutoff [3]. The Higgs mass is reliably computed in the effective theory, and is not dominated by unknown physics at the cutoff. However, the economy of the theory has been sacrificed. An entirely new sector of the theory must be introduced, with the sole purpose of breaking supersymmetry to generate the 
scale $m_{\mathrm{SUSY}}$. There are now many alternatives for this sector, and their relative merits are hotly debated, but the fact remains that such a new sector of the theory is inherent to the present formulation of supersymmetric theories. Indeed it was realized from an early stage that supersymmetry breaking could not occur in the standard model sector, but had to be somewhat remote from it [4], necessitating a mediation mechanism between the two sectors.

The result of this mediation is to introduce a set of new parameters describing the strengths of the soft supersymmetry breaking interactions. These parameters themselves give rise to a host of new problems: why are the squarks nearly degenerate so as to avoid flavor-changing and CP violating problems? Why are there any light Higgs bosons in the theory? There is no obvious symmetry keeping them light. Why is the proton stable? This success of the standard model is lost when the theory is made supersymmetric - apparently the proton could decay via squark exchange. What distinguishes matter from Higgs? In the standard model this is clear: matter is fermionic while Higgs is bosonic. Supersymmetry provides no such clear separation, requiring an artificial distinction between the Higgs boson and the sneutrino. Studying solutions to these problems has been an active area of research for many years.

Finally, one can ask how well supersymmetric theories account for the mass scale of the weak interactions, given that no superpartners have been discovered. The naturality of the proposed models is certainly not perfect: in gravity mediation only about $3 \%$ of parameter space gives acceptable theories, while in other schemes, such as gauge mediation, a similar amount of tuning is required to keep the charged slepton masses above the LEP2 limit.

On the other hand, supersymmetric theories with a perturbative energy desert certainly have some very positive features. It is possible to construct a relatively complete framework with a successful, precise prediction of the weak mixing angle [4, 5], the correct order of magnitude for neutrino masses from the see-saw mechanism [6], and the correct order of magnitude for the dark matter abundance from the cosmological freezeout of the lightest supersymmetric particle (LSP) [7]. However, given the shortcomings discussed above, we are motivated to investigate a more economical theory of EWSB.

Consider compact spatial dimensions, with a compactification scale $R^{-1}$ of order an inverse $\mathrm{TeV}$, in which standard model particles propagate [8]. In this case there are Kaluza-Klein (KK) towers for each particle propagating in this bulk. Imposing a symmetry on a compact space reduces the number of modes in the KK tower. This orbifold construction is crucial in obtaining chiral zero mode quarks, from a $5 \mathrm{~d}$ theory which is vector-like [9]. Furthermore, assume that the underlying bulk theory is supersymmetric. For example, if the top quark propagates in the bulk there will be KK towers for both the top quark and the top squark. In such a situation one can study radiative corrections to the Higgs boson mass with contributions from the entire KK towers [10]. In the case that supersymmetry is unbroken, there is an exact cancellation between the top and stop KK towers. However, if supersymmetry is broken so that the masses of the 
squark tower are shifted relative to the masses of the quark tower by an amount of order $R^{-1}$, the cancellation is no longer complete. Remarkably, the result is completely finite:

$$
m_{H}^{2} \propto-\left(\frac{1}{R}\right)^{2},
$$

and therefore independent of the cutoff, $\Lambda$, of the theory. The introduction of an extra compact dimension at the $\mathrm{TeV}$ scale allows a new resolution of the Higgs mass divergence - one where the physics of EWSB is necessarily right at the weak scale itself [10. Such Kaluza-Klein EWSB implies that the gauge couplings will become non-perturbative not far above the TeV scale [11] there is no perturbative energy desert - and fits well with the possibility that the fundamental scale of gravity is in the multi-TeV domain [12. What breaks supersymmetry, causing the mass shift between quark and squark KK towers?

Once extra dimensions have been introduced at the TeV scale, a new possibility opens up for supersymmetry breaking [8, 13], that is not available in the conventional energy desert version of supersymmetry: the Scherk-Schwarz mechanism [14]. Modifying the periodic boundary condition by using an $R$ symmetry, the excluded modes are different for fermions and bosons, breaking supersymmetry. An explicit extension of the standard model which breaks supersymmetry via the Scherk-Schwarz mechanism has been proposed [15], illustrating some important advantages over conventional supersymmetric theories. For example, the $\mu$ problem is solved, and supersymmetry breaking generates tree level Dirac masses for the gauginos and Higgsinos. However, a special choice of charges is necessary to keep the Higgs boson light at tree level. Below the compactification scale, $R^{-1} \simeq 25 \mathrm{TeV}$, a conventional, logarithmic radiative EWSB occurs, with a spectrum which is similar to gauge mediation. More generally, Scherk-Schwarz supersymmetry breaking offers the prospect of a predictive superpartner spectrum where radiative corrections are finite and dominated by the compactification scale [16]. This softness results because the supersymmetry breaking involves the global structure of the modes, and is therefore non-local. At distances beneath the compactification scale supersymmetry breaking effects are exponentially damped; this is quite unlike the case of supersymmetry in $4 \mathrm{~d}$, where radiative EWSB can originate from distances many orders of magnitude smaller than the weak scale. Given this exciting result, it is surprising that more explicit theories have not been constructed.

In this paper we construct a theory by combining Kaluza-Klein EWSB with non-local supersymmetry breaking. We introduce a single compact dimension of radius $R$ in which every particle of the standard model propagates.] The bulk of the $5 \mathrm{~d}$ theory is supersymmetric, so the KK

\footnotetext{
${ }^{1}$ Most studies of extra dimensions at the $\mathrm{TeV}$ scale have considered the case that the Higgs bosons propagate in the bulk, while the quarks and leptons do not 17, 18, 19, 20. This is surprising since the orbifold construction allows an elegant understanding of why the lightest bulk modes are chiral - a property which is crucial for matter but irrelevant for the Higgs. A possible reason for this is the power law running of couplings in 5d: with matter in the bulk the top Yukawa coupling grows more rapidly and the gauge couplings become non-perturbative before
} 
towers have multiplets corresponding to two supersymmetries in $4 \mathrm{~d}$. Two orthogonal reflection symmetries are imposed on the circle. One removes half of the modes in the KK towers, so that the zero modes possess a single supersymmetry. This zero mode structure is chiral, as needed for the quarks and leptons, but gives an electroweak gauge anomaly from the Higgsino. However, the second reflection symmetry reduces the number of supersymmetries to zero, yielding zero modes with precisely the states of the 1 Higgs doublet standard model. Supersymmetry breaking by this second reflection can be viewed as a discrete version [21] of the Scherk-Schwarz mechanism. Imposing one reflection symmetry to break one supersymmetry and obtain chiral zero modes has been widely used in the literature; the novel feature of our theory is the imposition of two reflections to break both supersymmetries. The bulk is remarkably pristine, containing just four parameters: the three gauge couplings and the compactification scale $R^{-1}$, which sets the mass scale for the KK towers. Every standard model particle is massless, and all the superpartners of the standard model have mass $R^{-1}$.

The physics of flavor occurs on branes not in the bulk. The reflection symmetries allow supersymmetric Yukawa interactions to be placed at their fixed points: for the up sector at the fixed point of one reflection, and for the down and charged lepton sectors at the fixed point of the second reflection. As the two reflection symmetries leave different supersymmetries unbroken, these Yukawa couplings involve the same Higgs doublet, even though the underlying theory is highly supersymmetric. These brane Yukawa interactions involve the usual 13 physical flavor parameters of the standard model. As in the case of conventional supersymmetry, there is no immediate progress in the understanding of flavor. Our theory is described in detail in section 2.

The Higgs boson interacts with the entire KK top quark tower on the branes containing the top quark Yukawa coupling, and Kaluza-Klein EWSB is induced. However, there are no extra free parameters to describe the resulting Higgs potential. Thus, the Fermi constant is used to determine the compactification scale, and the Higgs boson mass is predicted. The one-loop calculation of the compactification scale and the Higgs boson mass is presented in section 3 . In section 4 we show that the Higgs boson mass has very little sensitivity to unknown physics at short distances, although in the case of the compactification scale there is some sensitivity to the ultraviolet (UV). We also study the $\rho$ parameter and find that it has quadratic sensitivity to the UV.

Ignoring brane interactions, all superpartners are degenerate with mass $R^{-1}$. The large top Yukawa not only induces EWSB, but significantly modifies the spectrum of the top squarks, the neutralinos and the charginos. These masses are computed in section 5, where we show that the LSP is a top squark. The collider phenomenology of the superpartners is briefly discussed, and

they unify. We insist that all standard model particles propagate in the bulk, and find that there is an energy interval in which the 5 d theory is perturbative. Even with perturbative power law unification of gauge couplings, the prediction for the weak mixing angle is unreliable, as it is quadratically sensitive to the physics at the cutoff. 
we note that our theory possesses an anomaly free $U(1)_{R}$ symmetry. Conclusions are drawn in section 6 .

\section{The Theory and the Tree-level Spectrum}

We introduce a compact spatial dimension $y$, with a size of order an inverse $\mathrm{TeV}$, and require that the $5 \mathrm{~d}$ theory is supersymmetric. The standard model gauge bosons propagate in $5 \mathrm{~d}$ and are contained in $5 \mathrm{~d}$ vector supermultiplets $\left(A^{M}, \lambda, \lambda^{\prime}, \sigma\right)$. The 5 d vector field $A^{M}$ contains the standard model gauge boson from its first 4 components, and a $4 \mathrm{~d}$ scalar $A^{5}$. There are two gauginos, $\lambda$ and $\lambda^{\prime}$, reflecting the presence of two supersymmetries on reduction to $4 \mathrm{~d}$, and a real scalar $\sigma$. It is often convenient to view the theory as a $N=14 \mathrm{~d}$ theory; the $5 \mathrm{~d}$ vector multiplet can be decomposed into a 4 d vector supermultiplet $V\left(A^{\mu}, \lambda\right)$, and a chiral multiplet in the adjoint representation $\Sigma\left(\phi_{\Sigma}, \psi_{\Sigma}\right)$, where $\phi_{\Sigma}=\left(\sigma+i A^{5}\right) / \sqrt{2}$ and $\psi_{\Sigma}=\lambda^{\prime}$.

The standard model matter and Higgs fields also propagate in $5 \mathrm{~d}$, and are described by a set of hypermultiplets $\left(\Psi, \phi, \phi^{\prime}\right)_{X}$, where $\Psi$ is a Dirac fermion, and $\phi, \phi^{\prime}$ are two complex scalars. These each decompose into two $4 \mathrm{~d} N=1$ chiral multiplets $X\left(\phi_{X}, \psi_{X}\right)$ and $X^{c}\left(\phi_{X}^{c}, \psi_{X}^{c}\right)$, where $\Psi=\left(\psi, \psi^{c \dagger}\right)$ and $\phi^{\prime}=\phi^{c \dagger}$. Conjugated objects have conjugate transformations under the gauge group, and $\mathrm{X}$ runs over three generations of matter, $Q, U, D, L, E$, and a single Higgs $H$.

The 5 d theory contains a $S U(2)_{R}$ symmetry under which $\left(\lambda, \lambda^{\prime}\right)$ and $\left(\phi, \phi^{\prime}\right)$ are doublets, while all other fields are singlets. The two supersymmetries of the $4 \mathrm{~d}$ theory are related by $\lambda \leftrightarrow \lambda^{\prime}$ and $\phi \leftrightarrow \phi^{\prime}$ and play a crucial role in the construction of our theory. The $4 \mathrm{~d} N=1$ language, which we use from now on, hides both the $S U(2)_{R}$ symmetry and the second supersymmetry. The $N=1$ fields depend on $y$ as a continuous parameter; on compactification this leads to KK towers of $N=1$ fields.

\subsection{The $S^{1} / Z_{2}$ orbifold}

If the extra dimension is taken to be a circle, $S^{1}$, of radius $R$, then each field has modes $e^{ \pm i n y / R}$, $n=0,1,2, \ldots$, and the fermionic matter and Higgsino states are vectorial. To obtain chiral matter, we first consider restricting the space of the extra dimension to the orbifold $S^{1} / Z_{2}$. A $Z_{2}$ symmetry is introduced under which $y \rightarrow-y$ and all fields are either even or odd, having modes

$$
\begin{array}{cc}
+: & \cos \frac{n y}{R} \\
-: & \sin \frac{n y}{R}
\end{array}
$$

respectively, so that only the even fields contain zero modes. Since $A^{\mu}$ appears in the covariant derivative $D^{\mu}$ the vector multiplet is even, $V_{+}$. On the other hand $\operatorname{Im} \phi_{\Sigma}$ appears in $D^{5}$, and 
therefore the chiral adjoint multiplet is odd, $\Sigma_{-}$. The term in the superpotential $X D^{5} X^{c}$ ensures that $X$ and $X^{c}$ have opposite $Z_{2}$ charges. The conjugate label is arbitrary, and without loss of generality we may choose $X_{+}, X_{-}^{c}$ — the conjugate fields do not contain zero modes. These assignments are the same nomatter whether $X$ is matter or Higgs - the orbifold only has one type of hypermultiplet and does not provide any distinction between matter and Higgs. The physical space of the orbifold is the interval $0<y<\pi R$, since once a field configuration is specified in this region, it is fixed everywhere on the circle. The end points of the interval are fixed points under the $y \rightarrow-y$ transformation. The orbifolding has therefore broken $N=2$ to $N=1$ supersymmetry, and $5 \mathrm{~d}$ Lorentz to $4 \mathrm{~d}$ Lorentz symmetry at the boundary.

The symmetries of the bulk forbid interactions which break the flavor group $U(3)^{5}$ acting on $Q, U, D, L, E$. In particular, bulk Yukawa interactions are forbidden by supersymmetry. However, we can introduce interactions on the $4 \mathrm{~d}$ subspaces of the orbifold fixed points, which we call brane interactions. Such interactions necessarily violate 5 d Lorentz symmetry and $N=2$ supersymmetry. However, we insist that they preserve the same spacetime symmetries as the orbifold: $Z_{2}$, 4d Lorentz symmetry and $N=1$ supersymmetry. Note that we started with two supersymmetries, and we could have the orbifold and brane interactions break different supersymmetries. This would give a theory with supersymmetry completely broken in a hard way, and hence we require that the $Z_{2}$ orbifold and brane interactions leave one supersymmetry unbroken.

The allowed brane superpotential depends on the hypercharge sign choice for the Higgs chiral multiplet, $H$, which contains the zero mode. One choice allows $Q U H$, while the other allows $(Q D H+L E H)$ - we can get masses for the up sector, or for the down and charged lepton sectors, but not for both.'] One can take various views on this problem: perhaps it is not a problem, but a useful zeroth-order approximation allowing an understanding of why $m_{t} \gg m_{b}$, although the anomalies of the zero modes need to be cancelled. Alternatively, one could introduce two Higgs hypermultiplets, so that both choices can be made. This will lead to an minimal supersymmetric standard model (MSSM)-like 4d theory at scales below $1 / R$. In this case, the extra dimension has not addressed many of the issues familiar from the MSSM: supersymmetry breaking and mediation, the distinction between matter and Higgs and the origin of $R$ parity. In this paper we explore a third alternative.

We have seen that orbifolding with one $Z_{2}$ breaks one supersymmetry and allows one type of Yukawa coupling. The freedom of choice of the charge of $H$ turns out to be equivalent to the choice of which supersymmetry is kept unbroken by the orbifolding. We find that if we orbifold twice, using two $Z_{2}$ s, both supersymmetries can be broken, and both types of Yukawa coupling are allowed. The $Z_{2}$ s have different fixed points so that the two supersymmetries are broken at

\footnotetext{
${ }^{2}$ Brane interactions involving $X^{c}$ fields are also allowed by gauge invariance, but all components of $X^{c}$ vanish on the orbifold fixed points, and hence these interactions also vanish. $R$-parity violating interactions, such as $L L E$, are also allowed.
} 


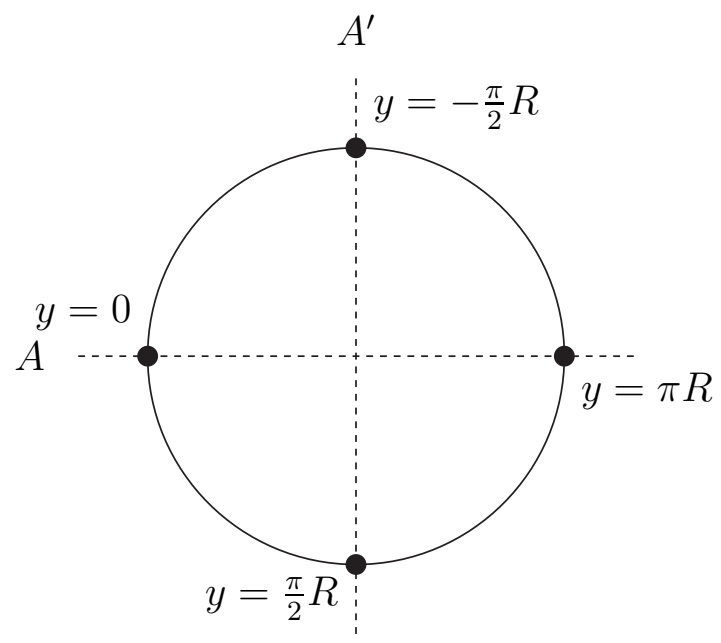

Figure 1: $S^{1} /\left(Z_{2} \times Z_{2}^{\prime}\right)$ orbifold in the fifth dimension.

different locations in the bulk, maintaining the softness of radiative corrections.

\subsection{The $S^{1} /\left(Z_{2} \times Z_{2}^{\prime}\right)$ orbifold}

The $S^{1} /\left(Z_{2} \times Z_{2}^{\prime}\right)$ orbifold is constructed from the circle by imposing two parities: $Z_{2}: y \rightarrow-y$ and $Z_{2}^{\prime}: y^{\prime} \rightarrow-y^{\prime}$, where $y^{\prime}=y-\pi R / 2$. These correspond to reflections about the axes $A$ and $A^{\prime}$ in Figure 1. The modes of the circle are now assembled into 4 types rather than 2 , according to their $\left(Z_{2}, Z_{2}^{\prime}\right)$ quantum numbers:

$$
\begin{array}{ll}
(+,+): & \cos \frac{2 n y}{R} \\
(+,-): & \cos \frac{(2 n+1) y}{R} \\
(-,+): & \sin \frac{(2 n+1) y}{R} \\
(-,-): & \sin \frac{(2 n+2) y}{R}
\end{array}
$$

with $n=0,1,2, \ldots$. Any component field will have just one type of mode, according to its $Z_{2} \times Z_{2}^{\prime}$ assignment; only fields with $(+,+)$ assignment contain a zero mode. The modes are completely specified over the circle once they are given on the interval $0<y<\pi R / 2$, which we choose to be the physical space.

There are two ways to interpret the quantum numbers of the two discrete $Z_{2}$ symmetries. One way is the following. The first $Z_{2}$, which is the reflection $y \rightarrow-y$, leaves a supersymmetry 

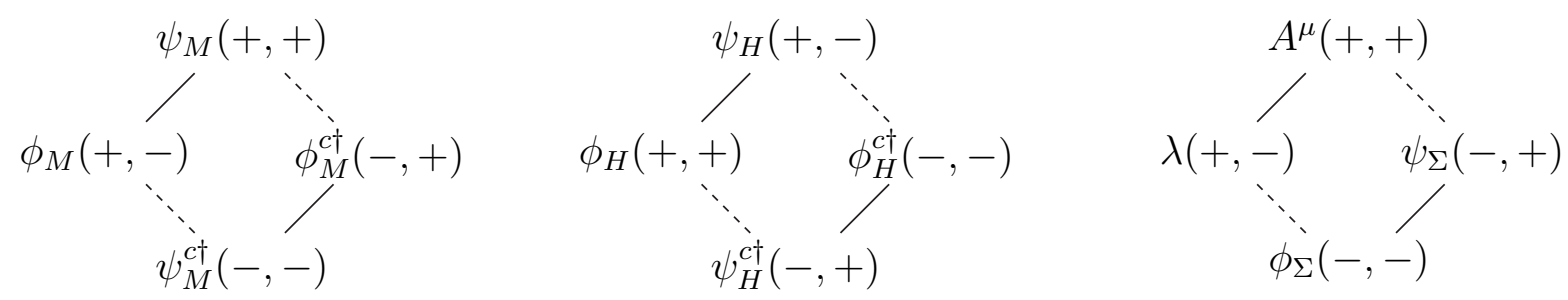

Figure 2: Quantum numbers of the matter, Higgs and gauge multiplets under the two orbifoldings $y \rightarrow-y$ and $y^{\prime} \rightarrow-y^{\prime}$.

$S$ unbroken, and gives $N=1$ multiplets with the usual $Z_{2}$ orbifold quantum numbers discussed in the previous sub-section, such as $X_{+}$and $X_{-}^{c}$. Brane interactions, located at the fixed points at $y=0, \pi R$, should preserve $S$. For example, choosing $H$ to have positive hypercharge allows the superpotential term $Q U H$. When $Z_{2}^{\prime}$ is introduced, the couplings of this interaction on the two branes at $y=0$ and $y=\pi R$ are constrained to be equal.

How does $Z_{2}^{\prime}$ act on these $N=1$ multiplets? This action is identified with the $R$ parity, $R_{P}$, of this $S$ supersymmetry, i.e. with $\theta \rightarrow-\theta$. For any hypermultiplet, $X$ can be chosen to be $R_{P}$ even or odd - there are now two types of hypermultiplet, one in which the zero mode is a scalar, and the other where the zero mode is a fermion. Thus supersymmetric theories on the $S^{1} /\left(Z_{2} \times\right.$ $Z_{2}^{\prime}$ ) orbifold provide an inherent distinction between Higgs and matter multiplets depending on whether their $Z_{2}$ and $Z_{2}^{\prime}$ quantum numbers are the same or different: $H(+,+), M(+,-)$. Invariance of the interactions $H D^{5} H^{c}$ and $M D^{5} M^{c}$, then, determines the quantum numbers for the conjugate multiplets $H^{c}(-,-), M^{c}(-,+)$. Covariance of the gauge derivatives $D^{\mu}$ and $D^{5}$ determines the assignments for the vector and chiral adjoint multiplets: $V(+,+), \Sigma(-,-)$. Making the usual superfield expansions, $H=\phi_{H}+\theta \psi_{H}$, etc, gives the quantum numbers for the components of the Higgs, matter and vector hypermultiplets shown in Figure 2.

It is interesting to note that anomaly freedom of the low energy effective theory does not allow a single Higgs hypermultiplet with a circle reduced by a single $Z_{2}$ orbifolding. Two $Z_{2}$ orbifoldings are necessary to restore anomaly freedom. For matter hypermultiplets, however, fermionic zero modes remain after orbifolding with both $Z_{2} \mathrm{~s}$ - anomalies cancel between multiplets as in the standard model.

The second orbifold clearly breaks supersymmetry, since the components of an $N=1$ superfield have different modes. The tree level spectrum is shown in Figure 3. The zero modes are in one-to-one correspondence with the particles of the standard model, and have KK excitations with masses $2 n / R$. The superpartners of standard model particles, and the states obtained by an $S U(2)_{R}$ transformation on these superpartners, have KK towers of states of mass $(2 n+1) / R$. 


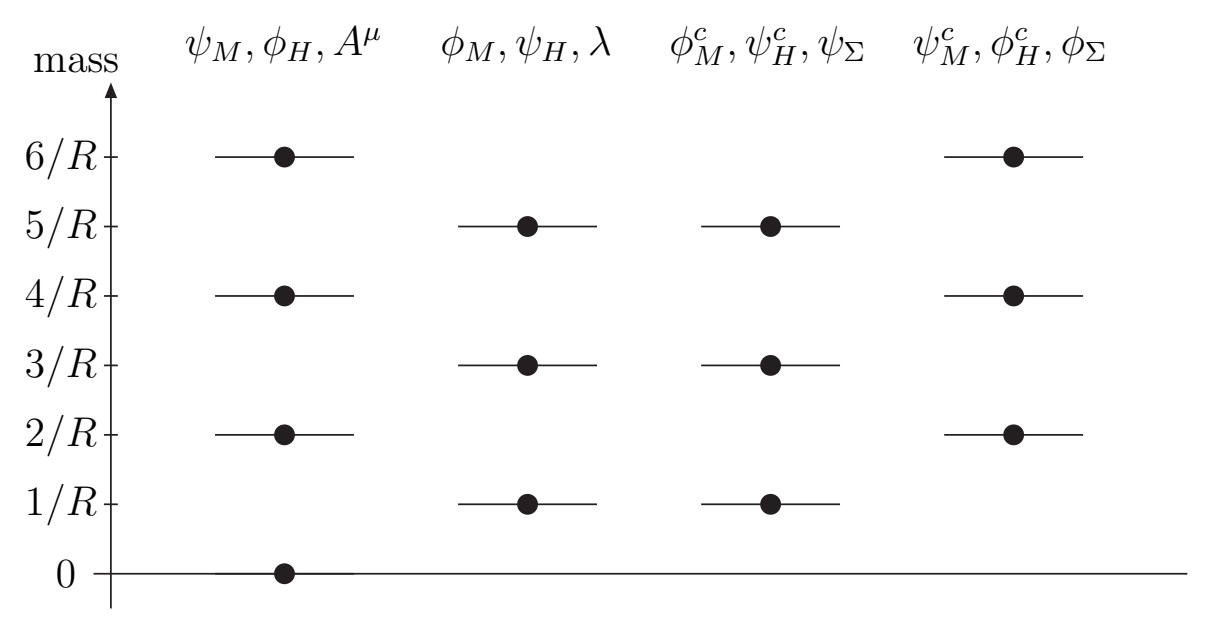

Figure 3: Tree-level KK mass spectrum of the matter, Higgs and gauge multiplets.

Finally, the conjugate quarks, conjugate Higgs and $\phi_{\Sigma}$ have KK towers of mass $(2 n+2) / R$. At an arbitrary point in the bulk, supersymmetry is also broken by the wavefunctions of the modes. At the orbifold fixed points at $y=0$ and $y=\pi R / 2$, the supersymmetry of the wavefunctions is restored, except for the zero mode.

The second interpretation of the $\left(Z_{2}, Z_{2}^{\prime}\right)$ quantum numbers results if we first orbifold the circle by $Z_{2}^{\prime}$ about the axis $A^{\prime}$ of Figure [1. This produces the same orbifold discussed in the previous sub-section, $S^{1} / Z_{2}^{\prime}$, with fixed points rotated by $\pi / 2$ to $y= \pm \pi R / 2$. The crucial point is that the supersymmetry left unbroken by this orbifolding, $S^{\prime}$, is not the supersymmetry $S$, which is preserved by first orbifolding by $Z_{2}$ about axis $A$. The $S^{\prime}$ multiplets are easily identified by grouping together the $Z_{2}^{\prime}=+$ and $Z_{2}^{\prime}=-$ component fields of Figure 2 :

$$
M^{\prime}\left(\begin{array}{c}
\phi_{M}^{c \dagger} \\
\psi_{M}
\end{array}\right) \quad M^{c \prime}\left(\begin{array}{c}
\phi_{M}^{\dagger} \\
\psi_{M}^{c}
\end{array}\right) \quad H^{\prime}\left(\begin{array}{c}
\phi_{H}^{c \dagger} \\
\psi_{H}
\end{array}\right) \quad H^{c \prime}\left(\begin{array}{c}
\phi_{H}^{\dagger} \\
\psi_{H}^{c}
\end{array}\right) \quad V^{\prime}\left(\begin{array}{c}
A^{\mu} \\
\psi_{\Sigma}
\end{array}\right) \quad \Sigma^{\prime}\left(\begin{array}{c}
\phi_{\Sigma} \\
\lambda
\end{array}\right) .
$$

The $S^{\prime}$ chiral superfields are labelled by the left-handed fermions they contain, while the $S^{\prime}$ vector multiplets are labelled by the bosonic components. The zero modes lie in $M^{\prime}, H^{c \prime}$ and $V^{\prime}$. On comparing with the $S$ multiplets

$$
M\left(\begin{array}{c}
\phi_{M} \\
\psi_{M}
\end{array}\right) \quad M^{c}\left(\begin{array}{c}
\phi_{M}^{c} \\
\psi_{M}^{c}
\end{array}\right) \quad H\left(\begin{array}{c}
\phi_{H} \\
\psi_{H}
\end{array}\right) \quad H^{c}\left(\begin{array}{c}
\phi_{H}^{c} \\
\psi_{H}^{c}
\end{array}\right) \quad V\left(\begin{array}{c}
A^{\mu} \\
\lambda
\end{array}\right) \quad \Sigma\left(\begin{array}{c}
\phi_{\Sigma} \\
\psi_{\Sigma}
\end{array}\right),
$$

one discovers that the transformation between $S$ and $S^{\prime}$ is accomplished by the interchanges $\left(\phi_{X} \leftrightarrow \phi_{X}^{c \dagger}\right)$ and $\left(\lambda \leftrightarrow \psi_{\Sigma}\right)$, a discrete subgroup of the $S U(2)_{R}$ symmetry. This interchanging between $S$ and $S^{\prime}$ multiplets is easily visualized from the multiplet arrangements of Figure 2 . 
The $\left(Z_{2}, Z_{2}^{\prime}\right)$ quantum numbers of both $S$ and $S^{\prime}$ superfields are also easily read from this Figure, as they are always given by the quantum numbers of the boson.

The brane interactions at the $Z_{2}^{\prime}$ fixed points $y= \pm \pi R / 2$ are $\left(Q^{\prime} D^{\prime} H^{c \prime}+L^{\prime} E^{\prime} H^{c \prime}\right)$. Remarkably, since $H^{c \prime}$ contains the zero mode Higgs boson, these are precisely the interactions needed to give mass to down-type quarks and charged leptons. Why is there not a complete symmetry under the interchange $Z_{2} \leftrightarrow Z_{2}^{\prime}$ and $S \leftrightarrow S^{\prime}$ ? The interchange $\left(\phi_{X} \leftrightarrow \phi_{X}^{c \dagger}\right)$ results in the zero mode Higgs boson lying in chiral multiplets of opposite hypercharge in the two cases, and this changes the form of the gauge-invariant brane interactions.

In this second viewpoint, $Z_{2}$ is identified as the $R$ parity, $R_{P}^{\prime}$, of the supersymmetry $S^{\prime}$, with $\theta^{\prime} \rightarrow-\theta^{\prime}$. Here $\theta^{\prime}$ is the superspace coordinate for $S^{\prime}$, for example: $M^{\prime}=\phi_{M}^{c \dagger}+\theta^{\prime} \psi_{M}$. This identification of $Z_{2}$ can be readily verified from the quantum numbers of Figure 2 .

The action of the discrete symmetries can be summarized by

$$
\begin{aligned}
& Z_{2}: \quad y \rightarrow-y, \quad\left(\theta^{\prime} \rightarrow-\theta^{\prime}\right)_{2}, \\
& Z_{2}^{\prime}: \quad y^{\prime} \rightarrow-y^{\prime}, \quad(\theta \rightarrow-\theta)_{1} .
\end{aligned}
$$

The superspace coordinates $\theta$ and $\theta^{\prime}$ are those of different orthogonal $N=1$ supersymmetry subgroups - they are not the superspace coordinates of the full $5 \mathrm{~d}$ theory. The first viewpoint uses the $\theta$ transformation, while the second viewpoint uses the $\theta^{\prime}$ ones. In the MSSM, $R$ parity is imposed in an ad hoc fashion to avoid proton decay - it is not inherent to the formulation of the theory. In our theory, $R$ parity becomes part of the orbifolding symmetry of (12), and is an unavoidable consequence of the basic formulation of the theory. It therefore comes as no surprise that, when the interactions of the low energy theory are derived, they are found to conserve baryon and lepton numbers at the renormalizable level.'?

Our theory may be formulated as a Scherk-Schwarz theory as follows. Start with a $S^{1} / Z_{2}$ orbifold of radius $R^{\prime}$, as described in the previous sub-section. On imposing the condition

$$
\phi\left(y+2 \pi R^{\prime}\right)=R_{P} \phi(y)
$$

on the modes of any component field $\phi$, one discovers that the allowed modes are precisely those of Eqs. (6- 9), with $R=2 R^{\prime}$. However, this formulation hides the symmetry between $Z_{2}$ and $Z_{2}^{\prime}$ symmetries, as it stresses the role of $Z_{2}$ and $R_{P}=Z_{2} Z_{2}^{\prime}$.

To summarize: we have taken every particle of the standard model to propagate in a compact dimension of size $R$. In the case that the $5 \mathrm{~d}$ theory is supersymmetric, and the compact space is the orbifold $S^{1} /\left(Z_{2} \times Z_{2}^{\prime}\right)$, the effective theory beneath the scale $1 / R$ is non-supersymmetric and chiral, having the gauge and multiplet structure of the standard model. The most general

\footnotetext{
${ }^{3} R$-parity violating interactions could be introduced, if we allow the coupling constants to have the opposite signs on two branes $y=0$ and $\pi R(y= \pm \pi R / 2)$.
} 
brane interactions, consistent with $Z_{2} \times Z_{2}^{\prime}$ and the supersymmetries preserved by each separate orbifold, up to cubic order, are

$$
\begin{aligned}
& \frac{1}{2}(\delta(y)+\delta(y-\pi R)) \int d^{2} \theta\left(\lambda_{U} Q U H\right) \\
+ & \frac{1}{2}\left(\delta\left(y-\frac{\pi}{2} R\right)+\delta\left(y+\frac{\pi}{2} R\right)\right) \int d^{2} \theta^{\prime}\left(\lambda_{D} Q^{\prime} D^{\prime} H^{c \prime}+\lambda_{E} L^{\prime} E^{\prime} H^{c \prime}\right)+\text { h.c. }
\end{aligned}
$$

Together these interactions break supersymmetry completely — but the locality of the operators results in the breaking being soft, even though the low energy 4 d theory contains $\psi_{Q} \psi_{U} \phi_{H}+$ $\psi_{Q} \psi_{D} \phi_{H}^{\dagger}$. At scales below $1 / R$, these give the Yukawa coupling matrices of the standard model proportional to the matrices $\lambda_{U, D, E}$. Hence, the low energy effective theory is precisely the standard model, with the Higgs potential constrained to have the tree-level form:

$$
V_{H, 0}=\frac{g^{2}+g^{2}}{8}\left|\phi_{H}\right|^{4}
$$

where $g$ and $g^{\prime}$ are the standard model $S U(2)$ and $U(1)$ gauge couplings. The absence of any free parameters in the Higgs potential at tree-level is striking. In the next section we calculate the radiative contributions to the Higgs potential from interactions involving the large top Yukawa coupling. We find that the effects of virtual KK towers leads at one loop to finite corrections to the Higgs potential involving the single parameter $R$. The Higgs mass-squared is negative, the Fermi constant is used to determine $R$, and the physical mass of the Higgs boson is predicted.

\section{The Higgs Boson Mass and the Compactification Scale}

In the previous section, we have investigated the tree-level structure of the model and found that the matter content of the massless sector is precisely that of the standard model. In this section, we calculate the one-loop effective potential of the Higgs boson coming from KK towers of the quark hypermultiplets through the top Yukawa coupling. We find that the Higgs boson receives

a negative mass-squared and EWSB is radiatively triggered. Furthermore, the effective potential is finite and depends only on the top Yukawa coupling $y_{t}$ and the compactification radius $R$. Thus, demanding the VEV of the Higgs field to be $175 \mathrm{GeV}$, we can calculate the value of the physical Higgs-boson mass and the compactification radius $R$, which determines the masses for the superpartners and the KK excitations.

\subsection{The Higgs mass squared}

Before computing the one-loop effective potential, we first calculate diagrammatically the masssquared for the Higgs doublet by making a KK decomposition of the original 5d theory. The 
interactions between the Higgs boson and the KK modes of the quark fields are read off by expanding the brane interaction given in Eq. (14). After eliminating the auxiliary $F$ fields, the relevant terms are

$$
\begin{aligned}
S_{\text {int }}= & \int d^{4} x\left[\sum_{k, l=0}^{\infty}\left(\frac{f_{t}}{\sqrt{2}} m_{\phi^{c}, k} \eta_{k}^{F} \eta_{l}^{\phi} \phi_{Q, k}^{c \dagger} \phi_{U, l} \phi_{H}+\frac{f_{t}}{\sqrt{2}} m_{\phi^{c}, k} \eta_{k}^{F} \eta_{l}^{\phi} \phi_{U, k}^{c \dagger} \phi_{Q, l} \phi_{H}+\text { h.c. }\right)\right. \\
& -\sum_{k, l, m=0}^{\infty}\left(\frac{f_{t}^{2}}{2} \eta_{k}^{\phi} \eta_{l}^{\phi}\left(\eta_{m}^{F}\right)^{2} \phi_{Q, k}^{\dagger} \phi_{Q, l} \phi_{H}^{\dagger} \phi_{H}+\frac{f_{t}^{2}}{2} \eta_{k}^{\phi} \eta_{l}^{\phi}\left(\eta_{m}^{F}\right)^{2} \phi_{U, k}^{\dagger} \phi_{U, l} \phi_{H}^{\dagger} \phi_{H}\right) \\
& \left.-\sum_{k, l=0}^{\infty}\left(\frac{f_{t}}{\sqrt{2}} \eta_{k}^{\psi} \eta_{l}^{\psi} \psi_{Q, k} \psi_{U, l} \phi_{H}+\text { h.c. }\right)\right],
\end{aligned}
$$

where $\phi_{H}$ is the zero-mode Higgs boson; $\phi_{M, k}, \psi_{M, k}, \phi_{M, k}^{c}$ and $\psi_{M, k}^{c}(M=Q, U)$ represent the $k$ th KK modes of the component fields in the hypermultiplet $M$ (see Figure 2). The dimensionless coupling $f_{t}$ is defined by $f_{t} \equiv\left(\lambda_{U}\right)_{33} /(\pi R)^{3 / 2} ; \eta_{k}^{\phi}, \eta_{k}^{\psi}$ and $\eta_{k}^{F}$ are the values of the wavefunctions at $y=0$ for the $\phi_{M, k}, \psi_{M, k}$ and $F_{M, k}$ fields, respectively.

The Higgs boson mass $m_{\phi_{H}}$ is generated at the one-loop level via loops of KK towers of the $Q$ and $U$ multiplets. There are three diagrams, as shown in Figure 4 , giving the contributions

$$
\begin{aligned}
-i m_{\phi_{H}}^{2}= & N_{c} f_{t}^{2} \sum_{k, l=0}^{\infty} \int \frac{d^{4} p}{(2 \pi)^{4}} \\
& \times\left\{\frac{\left(\eta_{k}^{F}\right)^{2}\left(\eta_{l}^{\phi}\right)^{2} m_{\phi^{c}, k}^{2}}{\left(p^{2}-m_{\phi^{c}, k}^{2}\right)\left(p^{2}-m_{\phi, l}^{2}\right)}-\frac{\left(\eta_{k}^{\psi}\right)^{2}\left(\eta_{l}^{\psi}\right)^{2} p^{2}}{\left(p^{2}-m_{\psi, k}^{2}\right)\left(p^{2}-m_{\psi, l}^{2}\right)}+\frac{\left(\eta_{k}^{\phi}\right)^{2}\left(\eta_{l}^{F}\right)^{2}}{\left(p^{2}-m_{\phi, k}^{2}\right)}\right\} .
\end{aligned}
$$

Thus, substituting the KK masses and wavefunctions obtained in the previous section,

$$
\left\{\begin{array} { l } 
{ m _ { \psi , k } = \frac { 2 k } { R } \equiv m _ { k } } \\
{ m _ { \phi , k } = m _ { \phi ^ { c } , k } = \frac { 2 k + 1 } { R } \equiv \tilde { m } _ { k } , }
\end{array} \quad \left\{\begin{array}{l}
\eta_{k}^{\phi}=\eta_{k}^{F}=1 \\
\eta_{k}^{\psi}=\left(\frac{1}{\sqrt{2}}\right)^{\delta_{k, 0}} \equiv \eta_{k},
\end{array}\right.\right.
$$

into Eq. (17), we obtain the one-loop induced Higgs boson mass-squared in the present model.

Performing a Wick rotation to Euclidian momentum space $p_{E}$, and changing to the variable $x=p_{E} R$, gives

$$
\begin{aligned}
-i m_{\phi_{H}}^{2}= & i N_{c} f_{t}^{2} \sum_{k, l=0}^{\infty} \int \frac{d^{4} p_{E}}{(2 \pi)^{4}} \\
& \times\left\{\frac{\tilde{m}_{k}^{2}}{\left(p_{E}^{2}+\tilde{m}_{k}^{2}\right)\left(p_{E}^{2}+\tilde{m}_{l}^{2}\right)}+\frac{\eta_{k}^{2} \eta_{l}^{2} p_{E}^{2}}{\left(p_{E}^{2}+m_{k}^{2}\right)\left(p_{E}^{2}+m_{l}^{2}\right)}-\frac{1}{\left(p_{E}^{2}+\tilde{m}_{k}^{2}\right)}\right\} \\
= & \frac{i N_{c} f_{t}^{2}}{R^{2}} \int \frac{d^{4} x}{(2 \pi)^{4}} x^{2} \\
& \times \sum_{k, l=0}^{\infty}\left[\frac{\eta_{k}^{2} \eta_{l}^{2}}{\left(x^{2}+(2 k)^{2}\right)\left(x^{2}+(2 l)^{2}\right)}-\frac{1}{\left(x^{2}+(2 k+1)^{2}\right)\left(x^{2}+(2 l+1)^{2}\right)}\right] .
\end{aligned}
$$



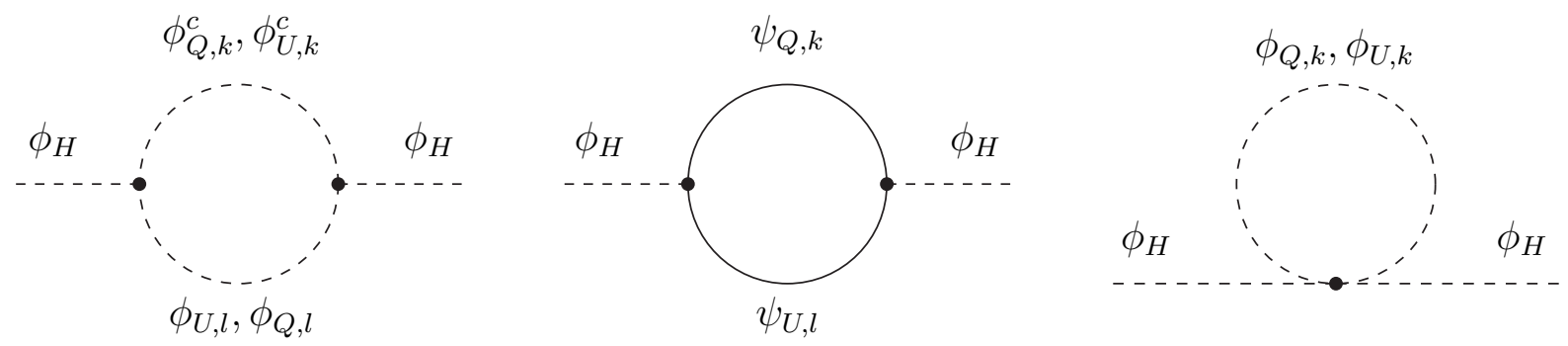

Figure 4: One-loop diagrams contributing to the mass squared of the Higgs boson.

In this expression, we first sum over the tower of KK states and then perform the momentum integral. The resulting Higgs mass-squared is

$$
\begin{aligned}
m_{\phi_{H}}^{2} & =-\frac{N_{c} f_{t}^{2}}{128 R^{2}} \int_{0}^{\infty} d x x^{3}\left\{\operatorname{coth}^{2}\left[\frac{\pi x}{2}\right]-\tanh ^{2}\left[\frac{\pi x}{2}\right]\right\} \\
& =-\frac{21 \zeta(3)}{64 \pi^{4}} \frac{N_{c} f_{t}^{2}}{R^{2}}
\end{aligned}
$$

where $\zeta(x)$ is the Riemann's zeta function. We find that the radiative correction $m_{\phi_{H}}^{2}$ is negative, so that EWSB is indeed triggered by loops involving the top KK towers. Furthermore, the result is finite and UV insensitive, since the momentum integral is exponentially cut off at $p_{E} \sim R^{-1}$; $99.99 \%(99 \%)$ of the integral comes from the region $p_{E} \lesssim 5 / R\left(p_{E} \lesssim 3 / R\right)$. This extreme softness arises because the geometrical separation between the two orbifold fixed points in the extra dimension acts as a point-splitting regularization. We can also rewrite Eq. (22) by using the $4 \mathrm{~d}$ top Yukawa coupling $y_{t}=f_{t} / 2^{3 / 2}$ as

$$
m_{\phi_{H}}^{2}=-\frac{21 \zeta(3)}{8 \pi^{4}} \frac{N_{c} y_{t}^{2}}{R^{2}} .
$$

It is interesting to note that a similar calculation for the squark mass-squared gives a vanishing result, $m_{\phi_{M}}^{2}=0$.

\subsection{The effective potential}

We would like to compute the Higgs potential with sufficient accuracy that the minimization leads to a determination of the compactification scale and the Higgs mass to better than $10 \%$. Balancing the negative Higgs mass squared, given above, against the tree-level gauge quartic Higgs interaction of Eq. (15) is not sufficient. In fact, it is not even sufficient to include the quartic interaction obtained from integrating out the top KK tower, as can be seen from the following 
simple argument. The only dimensionful parameter of our theory is $R$, so that this must set the scale of the effective potential. The contribution obtained by integrating out the top KK tower at one loop involves a factor of the top Yukawa coupling $y_{t}$ for each external Higgs field. Hence the one-loop top KK tower contribution takes the form $V_{t}=f(x) / R^{4}$, where $x=y_{t}^{2} R^{2} \phi_{H}^{\dagger} \phi_{H}$. Expanding $f(x)=A x+B x^{2}+C x^{2} \ln x+D x^{3}+\ldots$, all the coefficients, $A, B, C, \ldots$ arise at one loop and are expected to be comparable. Truncation at finite order is therefore unreliable.

The one-loop, all-orders, effective potential from integrating out the top KK tower is

$$
V_{t}(H)=\frac{1}{2} \operatorname{Tr} \int \frac{d^{4} p}{(2 \pi)^{4}} \sum_{k=-\infty}^{+\infty} \ln \left(\frac{p^{2}+m_{B_{k}}^{2}(H)}{p^{2}+m_{F_{k}}^{2}(H)}\right)
$$

where $H=\left|\phi_{H}\right|$ and the trace is taken over all states of the top hypermultiplet of a given $k$, giving a factor $4 N_{c}$. Clearly we only need the field dependent eigenvalues of the boson and fermion mass matrices $m_{B_{k}}$ and $m_{F_{k}}$. The top Yukawa coupling on the brane leads to a mixing of the tree level modes Eqs. (6-9) of the top states. For example, to leading order in $\left\langle\phi_{H}\right\rangle R$, the effective theory below $2 / R$ contains mass matrices for the squarks of the form

$$
-\mathcal{L}_{\text {mass }}=\left(\begin{array}{ll}
\phi_{Q, 0}^{\dagger} & \phi_{U, 0}^{c \dagger}
\end{array}\right)\left(\begin{array}{cc}
\left(\frac{1}{R}\right)^{2}+4 m_{t}^{2} & -2 m_{t} \frac{1}{R} \\
-2 m_{t} \frac{1}{R} & \left(\frac{1}{R}\right)^{2}
\end{array}\right)\left(\begin{array}{c}
\phi_{Q, 0} \\
\phi_{U, 0}^{c}
\end{array}\right)+(Q \leftrightarrow U) .
$$

However, this leading order matrix does not include exactly the effects of mixing between these states and the heavier states. We find the exact tree-level eigenvalue conditions to be

$$
\tan ^{2}\left(\frac{\pi R m_{F_{k}}}{2}\right)=\frac{\left(\pi y_{t} R H\right)^{2}}{4}
$$

and

$$
\cot ^{2}\left(\frac{\pi R m_{B_{k}}}{2}\right)=\frac{\left(\pi y_{t} R H\right)^{2}}{4}
$$

giving eigenvalues

$$
m_{B_{k}}(H)=\frac{2 k+1}{R} \pm m_{t}(H) \quad(k=0,1,2, \cdots)
$$

and

$$
m_{F_{k}}(H)=\frac{2 k}{R} \pm m_{t}(H) \quad(k=1,2,3 \cdots) .
$$

The zero-th order degeneracy of each level is split by $2 m_{t}$. At each mass eigenvalue there is a single Dirac fermion, or two complex scalars. There is also a single $k=0$ fermion mode, the top quark, with mass

$$
m_{t}(H)=\frac{2}{\pi R} \arctan \left(\frac{\pi y_{t} R H}{2}\right)
$$

4 Integrating out the tower of $\phi_{M, k}^{c}(k=1,2, \cdots)$ generates the term $\sum_{k=1}^{\infty}\left(f_{t}^{2} / 2\right) \phi_{M, 0}^{\dagger} \phi_{M, 0} \phi_{H}^{\dagger} \phi_{H}$ in the low-energy Lagrangian, which cancels infinities present in the bare Lagrangian Eq. (16), $-\sum_{k=0}^{\infty}\left(f_{t}^{2} / 2\right) \phi_{M, 0}^{\dagger} \phi_{M, 0} \phi_{H}^{\dagger} \phi_{H}$. 
As $H$ varies from 0 to $\infty, m_{t}(H)$ grows from 0 to a maximum value of $1 / R$. This dependence of the top quark mass on the electroweak field is quite unlike the standard model. For small $y_{t}$ it reduces to the standard model result $m_{t}=y_{t} H$, but, for the observed value of the top mass, the effect of mixings with the heavier KK modes is important. Increasing $H$ leads to a larger splitting of each level, but there is no level crossing. These eigenvalues can be used in Eq. (24), with $-\infty<k<+\infty$, by choosing the positive sign in Eqs. (28, 29), and, using calculational techniques from Ref. [16], we find

$$
V_{t}(H)=\frac{6 N_{c}}{\pi^{6} R^{4}} \sum_{k=0}^{\infty} \frac{\cos \left[(2 k+1) \pi R m_{t}(H)\right]}{(2 k+1)^{5}} .
$$

The denominator ensures that the higher terms in this series are rapidly suppressed. Taylor expanding around $H=0$, the quadratic term in this potential reproduces the mass-squared of Eq. (23) obtained by diagrammatic calculation.

The potential $V_{t}$ is a monotonically decreasing function of $H$, as shown in Figure 5. Runaway behaviour is prevented by the tree-level gauge potential $V_{H, 0}$ of Eq. (15), so that the combined potential $V_{H} \equiv V_{H, 0}+V_{t}$ has the minimum shown in Figure 5, given by the minimization condition

$$
\frac{1}{R}=\left(\frac{\pi^{6}}{18}\right)^{\frac{1}{4}}\left(M_{Z} v\right)^{\frac{1}{2}} \simeq 341 \mathrm{GeV},
$$

where $v=\langle H\rangle$. More precisely, the right-hand side should be multiplied by the factor $\xi^{-1 / 4}$ where

$$
\xi=\sin \left[\pi R m_{t}\right] \sum_{k=0}^{\infty} \frac{\sin \left[(2 k+1) \pi R m_{t}\right]}{(2 k+1)^{4}} .
$$

Accidentally, the minimum occurs at a value of $R$ such that $R m_{t}$ is very close to $1 / 2: \sin \left[\pi R m_{t}\right]$ is unity to better than $1 \%$, and the deviation of $\xi$ from unity only affects $1 / R$ at the level of about $1 \mathrm{GeV}$.

In the last section we stressed that compactification of our $5 \mathrm{~d}$ theory led to a low energy effective theory which was the standard model with the Higgs potential constrained to have the tree-level form of $V_{H, 0}$. In this section, we have discovered that EWSB in the low energy effective theory is quite unlike that of the standard model, because the relevant Higgs potential involves interactions to all orders in the Higgs field, not just the quadratic and quartic terms. For example, the Higgs VEV is not given by the familiar form $v^{2}=-m_{\phi_{H}}^{2} / \lambda$ where $\lambda$ is the quartic coupling. If this were true we would find $v^{2} \propto y_{t}^{2} R^{-2} /\left(g^{2}+g^{\prime 2}\right)$. Minimization of the all orders potential has given a different dependence:

$$
v^{4}=\frac{1}{\left(g^{2}+g^{\prime 2}\right)} \frac{L}{R^{4}},
$$




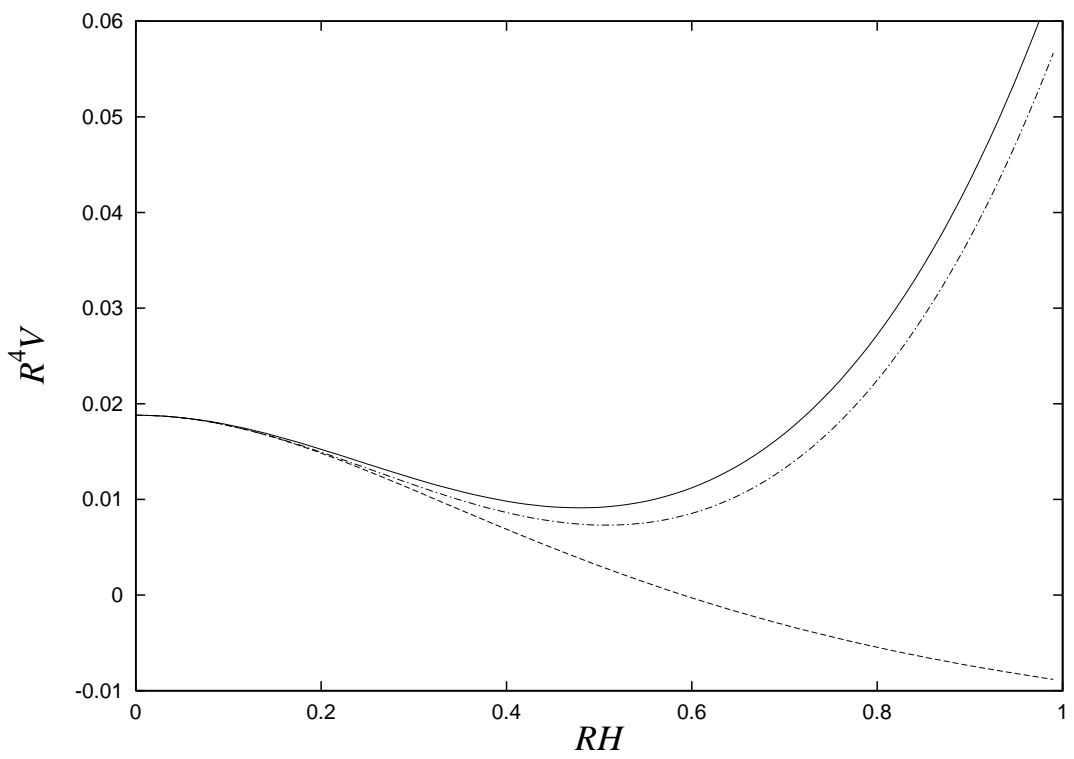

Figure 5: The Higgs potential $V$ normalized by the compactification radius $R$, as a function of $R H \equiv R\left|\phi_{H}\right|$. Dashed, dot-dashed and solid lines represent $V_{t}, V_{H} \equiv V_{H, 0}+V_{t}$ and $V_{H}$ with a gauge quadratic term, respectively.

where $L$ is the loop factor giving the relative size of tree and one-loop terms in the minimization condition $L=36 / \pi^{6}$. The scale of the $\mathrm{VEV}$ is still set by $R^{-1}$, and runaway is prevented by the non-zero value of the gauge couplings, but, because of the accident mentioned above, there is essentially no sensitivity to the uncertainty in the experimental value of the top quark mass.

Given that $R^{-1}$ is the only scale in the problem, why have the superpartners not yet been discovered? The origin of the difference between $R^{-1}$ and $v=175 \mathrm{GeV}$ can be seen from Eq. (34). The weakness of the gauge couplings actually increase $v$ above the compactification scale by $16 \%$. However, the loop factor $L^{1 / 4}$ increases $R^{-1}$ above $v$ by a factor of 2.3 . The weak gauge bosons are lighter than the superpartners because EWSB is driven only at one loop. Nevertheless, because it is the fourth root of $L$ that appears in $v$, the superpartner masses are not far above the weak boson masses.

Expanding $V_{H}$ about the minimum gives the physical Higgs mass

$$
m_{H}=\sqrt{2} M_{Z}\left(1-\frac{1}{4} \cos \left[\pi R m_{t}\right]\right) \simeq 127 \mathrm{GeV},
$$

where omitted terms in the expansion affect the Higgs mass by less than $1 \mathrm{GeV}$, and we have used a $\overline{\mathrm{MS}}$ top quark mass of $166 \mathrm{GeV}$ in evaluating $\cos \left[\pi R m_{t}\right] \simeq 0.06$. An uncertainty of 5 $\mathrm{GeV}$ in the experimental value of the top quark mass translates into a $2 \mathrm{GeV}$ uncertainty on the Higgs boson mass. 


\subsection{Predictions and uncertainties}

A correction to the effective potential of the Higgs boson comes from one-loop radiative corrections from the KK tower of the $S U(2) \times U(1)$ gauge multiplets. Here we study only the contribution to the quadratic term in the potential [16]

$$
\Delta m_{\phi_{H}}^{2}=\frac{21 \zeta(3)}{16 \pi^{4}} \frac{g^{2}+g^{\prime 2} / 3}{R^{2}} .
$$

The effect of including this quadratic term is shown in Figure 5. This increases $R^{-1}$ by $3 \%$.

The remaining uncertainties are the electroweak corrections to the higher terms in the effective potential and the two-loop top contributions to the effective potential, which we estimate at $1 \%$ and $6 \%$ respectively. We have calculated the induced VEVs for the higher KK modes of the Higgs boson, and found these effects to be at the $1 \%$ level. Hence the compactification scale is

$$
\frac{1}{R}=352 \pm 20 \mathrm{GeV},
$$

giving superpartner masses of $\simeq 350 \mathrm{GeV}$ and masses for the first $\mathrm{KK}$ excitations of $\simeq 700 \mathrm{GeV}$. The physical Higgs boson mass is

$$
m_{H}=127 \pm 8 \pm 2 \mathrm{GeV} .
$$

In these predictions, the first uncertainty is a combined theoretical uncertainty from the effects discussed above, while the second uncertainty in $m_{H}$ follows from the experimental uncertainty of $\pm 5 \mathrm{GeV}$ in the measured value of the top quark mass.

In the above calculation we performed summations over an infinite tower of KK modes. However, as discussed in the next section, our theory becomes strongly coupled at energies of about $5 / R$ and must be cutoff in some way. Are we correct to use the exact mass eigenvalues of Eqs. (28, 29) rather than the eigenvalues of mass matrices involving just the lower modes? Equivalently: what is the sensitivity of our results to the details of the UV cutoff? One may worry that there is a large sensitivity as each term in the sum of Eq. (20) is separately quadratically divergent. The crucial point is that we require our cutoff to preserve supersymmetry. Since supersymmetry is broken non-locally at the scale $R^{-1}$, the cutoff must preserve the cancellations which occur in the naive KK summation. This is non trivial, since a quadratic divergence still appears if the sums in Eq. (20) are terminated at some finite value, even keeping an equal number of bosonic and fermionic states. Thus we assume that the infinite sum over the KK modes gives the correct regularization of the theory; deviations from this "KK regularization" must be extremely small.

Finally, we note that in the above calculation it has been assumed that the KK towers of the quark multiplets have masses quantized precisely in units of $1 / R$. In general, however, the KK 
excitations receive wavefunction renormalizations due to brane interactions and their masses are renormalized. This can cause a shift of the compactification radius by as much as a few tens of percent, which thereby acquires some sensitivity to unknown UV physics. Remarkably, the Higgs mass prediction is much less sensitive to these wavefunction renormalizations, so that even in this case the tightness of Eq. (38) is maintained. This issue is discussed in the next section.

\section{Sensitivity to Physics above the Compactification Scale}

In the previous section we computed one-loop radiative corrections to the Higgs potential, yielding a prediction of both the Higgs boson mass and the compactification scale $1 / R$, which determines the masses of the superpartners and the KK excitations. The sum over KK modes softened the usual logarithmic divergence of supersymmetry to give a completely finite result, suggesting that the physics of EWSB is really governed by the energy scale $1 / R$, and has little sensitivity to whatever physics occurs at much higher energies. In this section we demonstrate that the compactification scale does have some sensitivity to physics in the UV, and we estimate this uncertainty. Remarkably, the Higgs mass is much less sensitive to these effects, so that the precise prediction of Eq. (38) is still expected to hold.

\subsection{Constraints from precision electroweak data}

For many theories in which the standard model gauge interactions propagate in a fifth dimension, precision electroweak data place a bound on the radius of order $R^{-1} \gtrsim 3 \mathrm{TeV}[19,20]$. These stringent bounds apply when matter or Higgs fields are located on a brane, and arise because the interactions of brane fields with the bulk gauge bosons violate momentum conservation in the extra dimension. For example, the kinetic term $\delta(y) Q^{\dagger} e^{g V} Q$ introduces interactions between the zero mode quarks and the excited modes of the gauge bosons: $\bar{q}_{0} \gamma_{\mu} A_{k}^{\mu} q_{0}$. This allows both the production of single gauge KK modes, $A_{k}^{\mu}$, and the generation of four zero-mode fermion operators from their virtual exchange. Similar effects result for brane leptons, while the situation for the brane Higgs is particularly dangerous. The operator $\delta(y) H^{\dagger} e^{g V} H$ causes mass mixing between the $W$ and $Z$ zero modes and their excited modes, and also induces a VEV for the weak triplet scalar in the $S U(2)$ chiral adjoint $\Sigma$ [20]. All these effects are absent to leading order in our theory because both matter and Higgs propagate in the bulk. All interactions, apart from the Yukawa interactions, conserve momentum in the fifth dimension, so that the effects that could produce the stringent bounds do not occur. Non-zero mode states can only be pair produced, and, for states of mass $1 / R \simeq 350 \mathrm{GeV}$, this will require further runs of the Tevatron and the LHC.

At one loop, the top Yukawa interaction generates brane kinetic terms for $Q_{3}, U_{3}$ and $H$. The 
contribution from short distances $(<R)$ is expected to be supersymmetric:

$$
\frac{1}{2}(\delta(y)+\delta(y-\pi R)) \int d^{4} \theta\left(Z_{Q_{3}} Q_{3}^{\dagger} e^{g V} Q_{3}+Z_{U_{3}} U_{3}^{\dagger} e^{g V} U_{3}+Z_{H} H^{\dagger} e^{g V} H\right) .
$$

Even though $Q_{3}, U_{3}$ and $H$ propagate in the bulk with kinetic terms $Q_{3}^{\dagger} e^{g V} Q_{3}$, etc, the brane kinetic interactions violate momentum conservation in the $y$ direction, leading to terms linear in KK modes. We define a dimensionless brane $Z$ factor $z_{X}=Z_{X} /(2 \pi R)$, so that, in the $4 \mathrm{~d}$ theory, the zero modes have kinetic terms with coefficient $1+z_{X}$. For $1 / R$ in the region of $350 \mathrm{GeV}$, experiments require $\left|z_{H}\right| \lesssim 0.2$. As we discuss below, $z_{H}$ is scale dependent - this bound applies at the scale $1 / R$. Limits on $z$ for the light quarks and leptons, from tree-level contributions of electroweak gauge bosons to precision electroweak observables, are also about 0.2. The experimental constraints on $z_{Q_{3}}$ and $z_{U_{3}}$ for the third generation quarks are much milder. We take this limit on the Higgs $Z$ factor to imply that it is also reasonable for these quark $Z$ factors to be less than 0.2 . This is strengthened by the estimates provided below, suggesting values of $z_{Q_{3}}$ and $z_{U_{3}}$ in the range of about 0.1 .

What are the effects of these $Z$ factors on the results of the calculation of the previous section? The $Z$ factor for the Higgs field does not affect the results at all. It is removed by going to canonical normalization before the calculation is begun, and affects only the relation between the $4 \mathrm{~d}$ and $5 \mathrm{~d}$ top coupling.

To find the effect of the $Z$ factors for $Q_{3}$ or $U_{3}$ we proceed as follows. We make a KK mode expansion and rescale the fields of the equivalent $4 \mathrm{~d}$ theory to obtain canonical kinetic energy. We study the mass matrices for the fermion and boson KK modes to linear order in $z_{Q, U}$, and find that the eigenvalue conditions have changed from Eqs. (26, 27) to

$$
\tan \left(\frac{\pi R m_{F_{k}}}{2\left(1-z_{Q}\right)}\right) \tan \left(\frac{\pi R m_{F_{k}}}{2\left(1-z_{U}\right)}\right)=\frac{\left(\pi R y_{t} v\right)^{2}}{4\left(1-z_{Q}\right)\left(1-z_{U}\right)}
$$

and

$$
\cot \left(\frac{\pi R m_{B_{k}}}{2\left(1-z_{Q}\right)}\right) \cot \left(\frac{\pi R m_{B_{k}}}{2\left(1-z_{U}\right)}\right)=\frac{\left(\pi R y_{t} v\right)^{2}}{4\left(1-z_{Q}\right)\left(1-z_{U}\right)},
$$

which, to linear order in $z_{Q, U}$, reduces to Eqs. (26, 27) with the replacement $1 / R \rightarrow(1-\bar{z}) / R$, where $\bar{z}=\left(z_{Q}+z_{U}\right) / 2$. With this replacement, Eqs. (28 - 35) all apply, so that it is $(1-\bar{z}) / R$ which is determined by the minimization of the Higgs potential to be $352 \mathrm{GeV}$. The numerical predictions for the top squark masses and Higgs are unaltered, while the other superpartner masses are

$$
\frac{1}{R} \simeq(1+\bar{z})(352 \pm 20) \mathrm{GeV}
$$

For $\left|z_{Q, U}\right|<0.2$, the uncertainty on $1 / R$ from $Z$ factors is $20 \%$. The Higgs and top squark masses are affected only at quadratic order in $z_{Q, U}$, which is at the percent level. 


\subsection{Power law running}

In the $5 \mathrm{~d}$ theory the gauge and Yukawa couplings display power law running behaviour. The $Z$ factor for the vector field, and therefore for the gauge coupling, is linearly divergent. From the $4 \mathrm{~d}$ viewpoint, the KK modes imply that the one-loop beta function coefficient at scale $E$ is proportional to $E R$, the number of KK modes lighter than $E$. For the chiral fields $Q_{3}, U_{3}$ and $H$, the $5 \mathrm{~d}$ interaction is proportional to $\delta(y)$, and this makes the $Z$ factor for these fields more divergent. From the 4d KK viewpoint, this can be seen directly from the one-loop wavefunction diagram. Instead of having a sum over a single tower of KK modes in the loop, as in the gauge case, there is a double sum, as the KK modes can be different in each propagator. Thus in the $4 \mathrm{~d}$ language, the coefficient of the anomalous dimension at energy $E$ is proportional to $(E R)^{2}$. At scale $1 / R, g_{3}$ and $y_{t}$ are comparable, but at larger energies the top coupling changes much more rapidly, and is the first coupling of the theory to become non-perturbative. Paying careful attention to the thresholds of the different species of particles, we find that the one-loop evolved top Yukawa coupling diverges at about 6/R. Using conventional strong coupling arguments, the top Yukawa coupling becomes non-perturbative at scale $M$ defined by

$$
y_{t}(M) \simeq \frac{4 \pi}{(M R)^{3 / 2}}
$$

Numerically, $M \simeq 5 / R \simeq 1.7 \mathrm{TeV}$. At this scale, the $4 \mathrm{~d}$ coupling $y_{t}$ may not be increased relative to its value at scale $1 / R$ by more than $20 \%$ or so. From the $4 \mathrm{~d}$ viewpoint the loss of perturbativity is largely due to the multiplicity of KK modes.

The gauge couplings change very little over the interval $1 / R$ to $5 / R$, and are all perturbative at $M$. At energies larger than $M$, evolving at one loop, the gauge couplings become nonperturbative before unifying — there is no calculable approximate power law unification. In fact, the one-loop analysis already becomes unreliable at $M$, as higher loop diagrams involve the non-perturbative coupling $y_{t}$.

\subsection{Estimate of brane $Z$ factors}

There are several reasons for studying the size of the brane $Z$ factors:

- The compactification scale has UV sensitivity only through the $Z$ factors.

- While the Higgs and top squark masses are remarkably insensitive to the $Z$ factors, small changes are possible.

- As the theory becomes non-perturbative at $M$, is it reasonable that $z_{H}(1 / R) \lesssim 0.2$ ?

- The degeneracy of many states at the scale $1 / R$ could be lifted by even quite small contributions to the brane $Z$ factors. 
We can consider two contributions to $Z_{H}$ - a boundary value at $M$, coming from the nonperturbative interactions above $M$, and a contribution that results from radiative corrections involving $y_{t}$ from $M$ to $1 / R$. Here we give rough estimates of these contributions.

Using a strong coupling analysis in higher dimensions [22, we estimate an upper bound for the boundary contribution of $Z_{H} \simeq 3 \pi /(2 M)$, giving $z_{H} \simeq 3 /(4 M R) \simeq 0.2$. We do not know that such boundary terms are present; however, even if this bound is saturated, there is no conflict with experiment - the effects in precision electroweak data are at the level of current observations. Of course, although not expected, we cannot exclude large boundary $Z$ factors for $Q_{3}$ and $U_{3}$ giving large shifts to the superpartner masses.

In scaling from $M$ to $1 / R$, the coupling $y_{t}$ changes by about $20 \%$. Using the one-loop formulae for the $Z$ factors, this scaling would generate $z_{H} \simeq 0.2$. Again we find a contribution at an acceptable but interesting level. Since this contribution gets most support from near $M$, where the theory approaches strong coupling, it is not surprising that our two contributions are estimated to be comparable. We stress that we do not know whether such contributions will be present in nature. Both are dominated by the UV, and, for example, it could be that our $5 \mathrm{~d}$ theory is cut off at some scale below $M$ by being incorporated into some other theory. Our analysis shows that large values of $z_{H}$ are not expected - our calculation of the compactification scale is under control and has limited sensitivity to the unknown UV physics, and the precise prediction of the Higgs mass is maintained.

Light fermions, such as the electron, do not have significant brane $Z$ factors generated by their Yukawa couplings. However, if our 5d theory is the low energy effective theory of some more fundamental theory, valid below some cutoff scale $\Lambda$, then the fundamental theory could lead to a value of the $Z$ factor at $\Lambda$, given purely be dimensional analysis, of $|Z| \simeq 1 / \Lambda$. In this case the first superpartner will have a mass shifted to $(1 / R)(1 \pm 1 /(2 \pi R \Lambda))$. This is a small effect - a $10 \mathrm{GeV}$ shift for $\Lambda=M$ - but represents an important lifting of the degeneracy. Brane kinetic terms for the gauge fields could similarly lift the degeneracy of the gauginos.

\subsection{The $\rho$ parameter}

The $\rho$ parameter has been measured to have the standard model value, with a limit on additional contributions $\Delta \rho<0.003$ at $95 \%$ confidence level, providing a powerful probe of new theories. Unfortunately the $\rho$ parameter cannot be reliably computed in our theory.

On first sight it appears that the dominant contribution to $\Delta \rho$ in our theory comes from the scalar states of the top quark hypermultiplets which have mass $1 / R$ in the absence of EWSB. By diagonalizing the $2 \times 2$ mass matrix of Eq. (25), we find that these states contribute $\rho^{(1)} \simeq 0.012$. If this were an accurate calculation of the dominant contribution to $\Delta \rho$, our theory would be experimentally excluded. However, it is not a reliable result for several reasons. If the "exact" eigenvalues of $1 / R \pm m_{t}$ are used we find $\rho^{(1)} \simeq 0.006$. If we include the " $3 / R$ " scalar states, by 
diagonalizing the relevant $4 \times 4$ scalar mass matrices, we find $\rho^{(1)}+\rho^{(3)} \approx 0.04$ using "exact" eigenvalues. Similarly the fermion states at $2 / R$ and $4 / R$ are expected to contribute $\rho^{(2)}+\rho^{(4)} \approx$ 0.02. These values are approximate as they are sensitive to the precise values used for the masses and mixings. We conclude that mixing between the lighter and heavier modes has an important effect on $\rho$.

The sensitivity of $\rho$ to higher modes is most dramatically seen by studying the contribution from the whole KK tower: a quadratic sensitivity to the UV emerges. We have already seen that radiative corrections from the top Yukawa interaction generate the interaction $\delta(y) \int d^{4} \theta Z_{H} H^{\dagger} e^{g V} H$ with $Z_{H}$ having a quadratic sensitivity to the cutoff due to the power law running of the top coupling. This $Z$ factor induces a positive contribution, $\rho_{\Sigma}$, from the VEV of the electroweak triplet in the $\Sigma$ multiplet. We now find that the higher dimension operator $\delta(y) \int d^{4} \theta\left(\xi / M^{4}\right)\left(H^{\dagger} e^{g V} H\right)^{2}$ is also generated with $\xi$ having quadratic sensitivity to the cutoff. In the strong coupling limit, this boundary operator could lead to a contribution as large as $\left|\rho^{M}\right| \simeq 0.05$. Contributions to the $\rho$ parameter are not reliably computed from the lowest lying KK mode. Our theory requires a cancellation between $\rho^{(1)}+\rho^{(2)}+\rho^{(3)}+\rho^{(4)}$ and the other contributions to $\Delta \rho$ at the level of one order of magnitude.

\section{Superpartner Spectrum and Collider Phenomenology}

What are the first states that will be encountered beyond those of the standard model? From Figure 3, we see that these are the superpartners, and their $S U(2)_{R}$ partners, which at tree level are all degenerate with mass $1 / R$. Consider the overall picture of the low energy effective theory beneath the TeV scale, after the states of mass $2 / R$ and above have been integrated out, so that only the standard model fields and the " $1 / R$ " states remain. It is clear that this effective theory is very different from the MSSM, which contains

- 2 Higgs doublets, and

- a single set of superpartners.

We stress that our effective theory below a $\mathrm{TeV}$ contains

- the 1 Higgs doublet standard model, and

- two superpartners for every standard model particle.

Even though our full theory is supersymmetric, our effective theory is not. There is no energy scale in which a $4 \mathrm{~d} N=1$ supersymmetric description is appropriate. Rather, the low energy theory reflects the underlying $N=2$ supersymmetry coming from the fifth dimension, with a superpartner $\tilde{p}$ and a conjugate superpartner $\tilde{p}^{c}$ for every standard model particle $p$

$$
p \Rightarrow\left(\tilde{p}, \tilde{p}^{c}\right)
$$




\begin{tabular}{|c||c|c|c|}
\hline$R$ & gauge $V$ & Higgs $H$ & matter $M$ \\
\hline+2 & & $h^{c}$ & \\
+1 & $\tilde{\lambda}$ & $\tilde{h}^{c}$ & $\tilde{m}, \tilde{m}^{c}$ \\
0 & $A^{\mu}, A^{c}$ & $h$ & $m, m^{c}$ \\
-1 & $\tilde{\lambda}^{c}$ & $\tilde{h}$ & \\
\hline
\end{tabular}

Table 1: Continuous $R$ charges for gauge, Higgs and matter components. Here, $m$ represents $q, u, d, l, e$.

In this section, we use a tilde to denote these superpartners: $\tilde{q}, \tilde{u}, \tilde{d}, \tilde{l}, \tilde{e}$ for the squarks and sleptons, $\tilde{h}$ for the Higgsino, and $\tilde{g}, \tilde{\omega}, \tilde{z}, \tilde{\gamma}$ for the gauginos, which we collectively denote by $\tilde{\lambda}$.

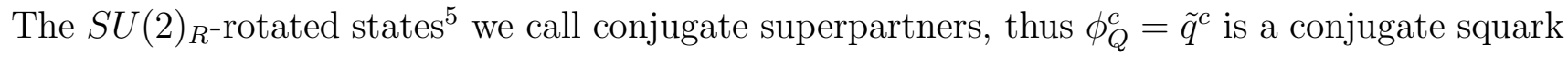
and $\psi_{\Sigma}$ of the $S U(3)$ gauge multiplet is a conjugate gluino, $\tilde{g}^{c}$.

\section{$5.1 \quad$ Spectrum}

In studying the mass matrices for these states, it is useful to notice that the theory possesses an accidental, continuous $R$ symmetry. We choose it to be such that $\theta\left(\theta^{\prime}\right)$ carries $R$ charge +1 $(-1)$. The resulting $R$ charges are shown in Table 1, and are the same for all members of the KK tower. The standard model particles are neutral, while the superpartners and their conjugates have charges \pm 1 . While the conjugate matter fields and conjugate gauge fields (i.e. the $\Sigma$ scalar) are neutral, the conjugate Higgs field has charge +2 . The absence of any $A$ terms or Majorana gaugino masses can be traced to this $R$ symmetry. The pattern of $R$ charges is not symmetrical about zero for the matter and Higgs fields because we insist that the Higgs $h$ is $R$ neutral, so that the $R$ symmetry is not spontaneously broken.

The leading effect which lifts the degeneracy of these states is EWSB. Even though this occurs radiatively, $v R \simeq 0.4$ is given by the 4 th root of the loop factor $L$, and is not small. These effects are important only for the top squarks and their conjugates, and for neutralinos and charginos.

There are four top-type scalars, but fortunately $U(1)_{R} \times U(1)_{E M}$ ensures the mass matrix splits into two $2 \times 2$ blocks. Since $\tilde{t}_{L} \subset \tilde{q}_{3}$ and $\tilde{t}_{R}=\tilde{u}_{3}^{\dagger}$, the two stop scalars $\tilde{t}_{L}$ and $\tilde{t}_{R}$ have opposite $R$ charges and cannot mix. Nevertheless, mixings can occur within the pair $\left(\tilde{t}_{L}, \tilde{t}_{R}^{c \dagger}\right)$ and the pair $\left(\tilde{t}_{R}, \tilde{t}_{L}^{c \dagger}\right)$. Each pair has the mass matrix given in Eq. (25). Taking the mixing with the heavier states into account, the eigenvalues are

$$
m_{ \pm}=\frac{1}{R} \pm m_{t}
$$

\footnotetext{
${ }^{5}$ The only case where $\tilde{p}$ and $\tilde{p}^{c}$ are not in the same $S U(2)_{R}$ doublet is the Higgsino.
} 


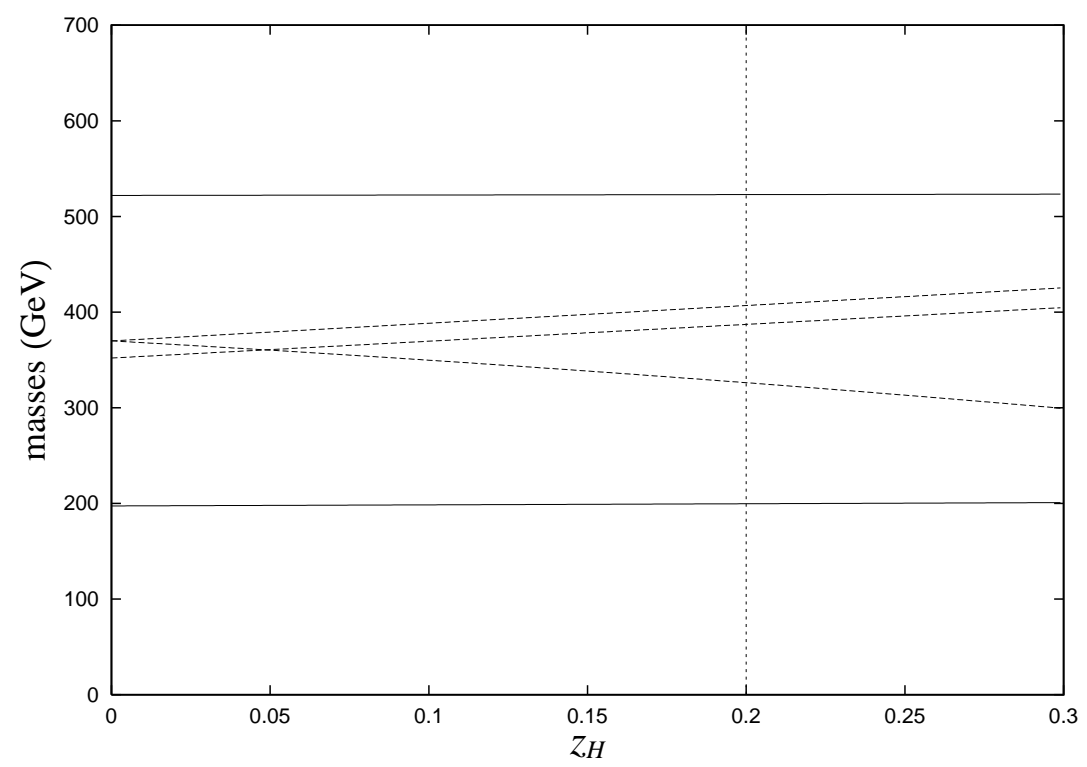

Figure 6: The top-squark and chargino masses as a function of $z_{H}$. Solid, dashed lines represent $\tilde{t}_{L, R_{+,-}}$and $\tilde{\chi}_{1,2,3}^{+}$fields, respectively. $z_{H}>0.2$ is disfavored by precision electroweak data.

For $R^{-1}=352 \mathrm{GeV}$, there are two charged $2 / 3$ colored scalars, $\tilde{t}_{L, R_{-}}$, of mass $186 \mathrm{GeV}$, and two, $\tilde{t}_{L, R_{+}}$, of mass $518 \mathrm{GeV}$. The $L, R$ subscript labels whether the state contains $\tilde{t}_{L}$ or $\tilde{t}_{R}$. Each mass eigenstate has roughly comparable $S U(2)_{L}$ doublet and singlet components, and each has a large coupling to the top quark and the Higgsino. Including one-loop radiative corrections from the QCD gauge KK tower increases these masses by $6 \%$ for $\tilde{t}_{-}$to $197 \mathrm{GeV}$ and $1 \%$ for $\tilde{t}_{+}$to 522 $\mathrm{GeV}$. Remarkably, the one-loop contribution proportional to the top coupling $y_{t}^{2}$ vanishes.

Introducing brane $Z$ factors for $Q_{3}$ and $U_{3}$, as in Eq. (39), the $2 \times 2$ mass matrix for the scalars $\left(\tilde{t}_{L}, \tilde{t}_{R}^{c \dagger}\right)$ becomes

$$
\left(\begin{array}{ll}
\tilde{t}_{L}^{\dagger} & \tilde{t}_{R}^{c}
\end{array}\right)\left(\begin{array}{cc}
\frac{1-2 z_{Q}}{R^{2}}+4 m_{t}^{2} & -2 m_{t} \frac{1-z_{U}}{R} \\
-2 m_{t} \frac{1-z_{U}}{R} & \frac{1-2 z_{U}}{R^{2}}
\end{array}\right)\left(\begin{array}{c}
\tilde{t}_{L} \\
\tilde{t}_{R}^{c \dagger}
\end{array}\right),
$$

to linear order in $z_{Q, U}$. The mass matrix for the scalars $\left(\tilde{t}_{R}, \tilde{t}_{L}^{\dagger \dagger}\right)$ is obtained by the interchange $z_{Q} \leftrightarrow z_{U}$. To leading order in $z_{Q, U}$ and in $m_{t}$ the eigenvalues of this matrix are $(1-\bar{z}) / R \pm m_{t}$, where $\bar{z}=\left(z_{Q}+z_{U}\right) / 2$. Using Eq. (41), which includes mixings with all heavier scalar modes, one discovers that this result is exact in $m_{t}$. As shown in section 3 , minimization of the Higgs potential determines $(1-\bar{z}) / R=352 \mathrm{GeV}$, so that the top squark masses are independent of the $Z$ factors, to linear order, as shown in Figure 6 .

Although there are six charginos and six neutralinos, the mass matrices have a very simple form, largely determined by $U(1)_{R}$ and $U(1)_{E M}$ symmetries. Both charginos and neutralinos 
have a $3 \times 3$ Dirac mass matrix, which splits into block diagonal form, giving chargino masses

$$
\left(\begin{array}{ll}
\tilde{h}^{+} & \tilde{\omega}^{c+}
\end{array}\right)\left(\begin{array}{cc}
\frac{1}{R} & \sqrt{2} M_{W} \\
-\sqrt{2} M_{W} & \frac{1}{R}
\end{array}\right)\left(\begin{array}{l}
\tilde{h}^{c-} \\
\tilde{\omega}^{-}
\end{array}\right)+\frac{1}{R} \tilde{\omega}^{c-} \tilde{\omega}^{+},
$$

and neutralino masses

$$
\left(\begin{array}{ll}
\tilde{h}^{0} & \tilde{z}^{c}
\end{array}\right)\left(\begin{array}{cc}
\frac{1}{R} & M_{Z} \\
-M_{Z} & \frac{1}{R}
\end{array}\right)\left(\begin{array}{c}
\tilde{h}^{c 0} \\
\tilde{z}
\end{array}\right)+\frac{1}{R} \tilde{\gamma}^{c} \tilde{\gamma} .
$$

The minus signs in the $(2,1)$ entries are extremely important. The pure gaugino states, $\tilde{\chi}_{1}^{+}$ and $\tilde{\chi}_{1}^{0}$, are the lightest, with mass $R^{-1}=352 \mathrm{GeV}$. The mixed gaugino/Higgsino states are increased in mass: $\tilde{\chi}_{2,3}^{+}$both have mass $R^{-1} \sqrt{1+2 M_{W}^{2} R^{2}}=370 \mathrm{GeV}$, and $\tilde{\chi}_{2,3}^{0}$ both have mass $R^{-1} \sqrt{1+M_{Z}^{2} R^{2}}=364 \mathrm{GeV}$.

These shifts are of order $M_{W}^{2} / R$ and hence small. The brane wavefunction $Z$ factor for the Higgs field does not correct the form of the $\tilde{\chi}_{1}$ masses, but does induce corrections to the form of the $\tilde{\chi}_{2,3}$ mass matrices, which, to linear order in $z_{H}$, become

$$
\left(\begin{array}{ll}
\tilde{h}^{+} & \tilde{\omega}^{c+}
\end{array}\right)\left(\begin{array}{cc}
\frac{1}{R}\left(1-z_{H}\right) & \sqrt{2} M_{W}\left(1+\frac{1}{2} z_{H}\right) \\
-\sqrt{2} M_{W}\left(1-\frac{1}{2} z_{H}\right) & \frac{1}{R}
\end{array}\right)\left(\begin{array}{c}
\tilde{h}^{c-} \\
\tilde{\omega}^{-}
\end{array}\right),
$$

for the charginos, and

$$
\left(\begin{array}{ll}
\tilde{h}^{0} & \tilde{z}^{c}
\end{array}\right)\left(\begin{array}{cc}
\frac{1}{R}\left(1-z_{H}\right) & M_{Z}\left(1+\frac{1}{2} z_{H}\right) \\
-M_{Z}\left(1-\frac{1}{2} z_{H}\right) & \frac{1}{R}
\end{array}\right)\left(\begin{array}{c}
\tilde{h}^{c 0} \\
\tilde{z}
\end{array}\right),
$$

for the neutralinos. The mass eigenvalues for the charginos are shown in Figure 6 as a function of $z_{H}$. The neutralino mass eigenvalues have a very similar behaviour, but with a smaller splitting at $z_{H}=0$. The $\tilde{\chi}_{1}^{+}$state has a mass $R^{-1}$, and changes with $z_{H}$ only because the extraction of $R^{-1}$ from the Higgs VEV involves $z_{H}$. The order $z_{H}$ terms in Eq. (49) cause a significant mass splitting between $\tilde{\chi}_{2}^{+}$and $\tilde{\chi}_{3}^{+}$, so that $\tilde{\chi}_{1}^{+}$may well not be the lightest chargino.

All the remaining superpartners, squarks, sleptons, gluinos and their conjugates, remain closely degenerate, with a mass of $R^{-1}$. Radiative corrections from the strong interaction lift the mass of the colored states by about $6 \mathrm{GeV}$ compared to the non-colored ones. EWSB allows us to determine $R^{-1}=352 \pm 20 \mathrm{GeV}$, where the uncertainty is from our theoretical calculation. Brane $Z$ factors can also affect $R^{-1}$ as shown by the $\tilde{\chi}_{1}^{+}$curve in Figure 6 , for the case that the $Z$ factors are generated purely from the 5 d power law running of the top coupling.

The supergravity multiplet in 5 d contains the graviton $G_{M N}$, the gravitino $\Psi_{M}$ and a vector field $B_{M}$. The KK decomposition of this $5 \mathrm{~d}$ multiplet leads to two $4 \mathrm{~d}$ multiplets: the graviton multiplet $\left(g_{\mu \nu}(+,+), \psi_{3 / 2}(+,-), \psi_{3 / 2}^{c}(-,+), B_{\mu}(-,-)\right)$ and the radion multiplet $\left(\left(g_{55}+\right.\right.$ $\left.\left.i B_{5}\right)(+,+), \psi_{1 / 2}(+,-), \psi_{1 / 2}^{c}(-,+), g_{\mu 5}(-,-)\right)$. The towers of KK modes have the usual spectrum with only the $4 \mathrm{~d}$ graviton and the radion $g_{55}$ having a zero mode, while the next lightest states are the fermionic ones at $1 / R$. We assume that the radion acquires a mass by a mechanism that 
stabilizes the size of the compact dimension. It has zero $R$ charge and is unstable to decay to standard model particles.

The two top scalars $\tilde{t}_{L-}$ and $\tilde{t}_{R-}$ are lighter than all other superpartners by a significant amount, having masses

$$
m_{\tilde{t}_{-}}=197 \pm 20 \mathrm{GeV} .
$$

This result is remarkably insensitive to brane $Z$ factors, as shown in Figure 6. The top scalars remain at least $100 \mathrm{GeV}$ lighter than the lightest chargino or neutralino. In addition, the brane $Z$ factors do not lift the degeneracy between $\tilde{t}_{L-}$ and $\tilde{t}_{R-}$ to the leading order. Since the one-loop gauge correction and $D$-term contribution also do not lift it, the mass splitting between these two scalars would be, if any, very small.

Although $\tilde{t}_{L-}$ and $\tilde{t}_{R-}$ have opposite $U(1)_{R}$ charges, the heavier may decay to the lighter, for example by $\tilde{t}_{L_{-}} \rightarrow \tilde{t}_{R-}^{\dagger} u u$. This decay could be mediated by a flavor-changing gluino exchange, and the lifetime is very sensitive to the scalar mass differences and the size of the flavor changing coupling. Decays may also be induced by certain higher dimension operators. For the most plausible ranges of parameters the decay does not occur inside a collider detector, unless the heavier scalar is stopped, but would be expected to occur cosmologically. We refer to these two scalars as the LSP and the next to LSP (NLSP).

\subsection{Collider phenomenology}

For run II of the Fermilab collider the most promising signals of our theory are the standard model Higgs of mass at $127 \pm 8 \mathrm{GeV}$, and the LSP and NLSP top scalars of mass $197 \pm 20 \mathrm{GeV}$. The pair production cross section for these scalars at a center of mass energy of $2 \mathrm{TeV}$ is $360 \mathrm{fb}$ each [23]. This is significantly more than the rate for producing them via pair production of the gluinos and other squarks with mass of $350 \mathrm{GeV}$, followed by cascade decays to the LSPs. Once pair produced, these scalars will hadronize by picking up a $u$ or $d$ quark and becoming a fermionic meson $T^{0}=\bar{u} \tilde{t}_{-}$or $T^{+}=\bar{d} \tilde{t}_{-}$, with almost equal probability. While the charged meson is expected to be slightly heavier than the neutral one, both will be sufficiently stable to traverse the entire detector. Hence the signals for scalar top pair production are events with one or two heavy stable particles with electric charge \pm .9 Furthermore, the anti-stop bound states, $\bar{T}^{0}=u \overline{\tilde{t}}_{-}$and $T^{-}=d \overline{\tilde{t}}_{-}$, can oscillate by exchanging isospin and charge with background material in the detector, causing intermittent highly ionizing tracks. These signals have been investigated in the context of gauge mediation models with stop NLSP 24]. The present experimental limit on such particles from Run Ib at the Tevatron collider is about $150 \mathrm{GeV}$ from CDF [25].

${ }^{6}$ If $R$-parity violating interactions are introduced on the branes, the top scalars may decay into the standard model particles inside the detector. 
For sufficiently low speed $\beta$, the charged mesons $T^{+}$will stop inside the detector, and eventually give a positron with very low momentum, presumably in the MeV range, by $\beta$ decay to the neutral state. If $T^{+}$contains the NLSP, another possibility is that this NLSP decays to the LSP giving decay products with energies of order the scalar mass difference, for example in the $\mathrm{GeV}$ range.

At the LHC the situation is more complicated since all the squarks, gluinos and their conjugates will be pair produced. However, these will all cascade to the LSP or NLSP, so once again a crucial signal becomes the observation of events with one or two stable charged particles. The initial pair production reaction produces one particle with $U(1)_{R}$ charge +1 , and one with -1 . These cascade to eventually give $\tilde{t}_{L-}$ or $\tilde{t}_{R-}^{\dagger}$ and $\tilde{t}_{R-}$ or $\tilde{t}_{L-}^{\dagger}$ respectively. Thus, unlike in the case of direct scalar top production, events occur in which the stable charged particles have the same sign.

If the gluino is heavier than the squarks the decay chain is $\tilde{g} \rightarrow q \tilde{q}$ and $\tilde{q} \rightarrow q \tilde{\chi}$, where $q$ and $\tilde{q}$ refer to any flavor except top, when the decay is not forbidden by phase space. For the case that the squarks are heavier than the gluino the decays are $\tilde{q} \rightarrow q \tilde{g}$ and $\tilde{g} \rightarrow q \bar{q} \tilde{\chi}$. Recall that $\tilde{g}$ and $\tilde{\chi}$ are Dirac fermions, and $\tilde{q}$ refers to squarks and conjugate squarks. All $\tilde{\chi}$ states that are kinematically open will be populated. The three charginos will all decay via $\tilde{\chi}^{+} \rightarrow \bar{b} \tilde{t}_{-}$. The neutralinos decay to $\bar{t} \tilde{t}_{-}$if open, but the lightest neutralino will decay via a virtual chargino: $\tilde{\chi}^{0} \rightarrow \bar{q} q \tilde{\chi}^{+}, \tilde{\chi}^{+} \rightarrow \bar{b} \tilde{t}_{-}$. Hence all events resulting from pair production of the " $1 / R$ " states contain two $b$ quark jets.

\section{$5.3 \quad \mathrm{U}(1)_{R}$ symmetry}

In subsection 5.1, an accidental $U(1)_{R}$ symmetry, given in Table 1, played an important role to reveal the mass spectrum of the theory. Here we note that this $U(1)_{R}$ is an anomaly-free symmetry. In the usual $4 \mathrm{~d} N=1$ theory, an anomaly free $U(1)_{R}$ symmetry requires the introduction of additional exotic states, beyond those of the MSSM, since the gauginos and the gravitino carry a $U(1)_{R}$ anomaly 26. However, in the present model, there are conjugate gauginos and conjugate gravitino, so that the $U(1)_{R}$ is automatically anomaly free; the quarks and leptons have zero charges and all the other fermionic states are vector-like. The $U(1)_{R}$ symmetry is a linear combination of the $U(1)$ subgroup of the $S U(2)_{R}$ automorphism group of $N=2$ supersymmetry algebra and a vector-like, non- $R U(1)$ symmetry under which the Higgs fields transform as $H(-1)$ and $H^{c}(+1)\left(H^{\prime}(-1)\right.$ and $\left.H^{c \prime}(+1)\right)$ again demonstrating that $U(1)_{R}$ is anomaly free.

The above remarkable property allows us to impose the $R$ symmetry as an exact symmetry of the theory. Since neither EWSB $\langle h\rangle \neq 0$ nor chiral condensation $\langle q \bar{q}\rangle \neq 0$ breaks this $U(1)_{R}$ symmetry, it may remain as an unbroken symmetry. Then, the LSP is absolutely stable since the $R$ parity, $R_{P}$ or $R_{P}^{\prime}$, is a discrete subgroup of the $U(1)_{R}$ symmetry, and some higher-dimensional operators are forbidden by the symmetry. 
Anomaly freedom raises the interesting possibility that this $U(1)_{R}$ symmetry is a gauge symmetry. In the usual $4 \mathrm{~d} N=1$ supergravity, a gauged $R$ symmetry cannot remain at low energies since a non-vanishing Fayet-Iliopoulos $D$ term of order the Planck scale is generated and breaks $U(1)_{R}$ at the Planck scale [27]. This is true even in the $N=2$ case [28]. If the same is true of our theory, the low-energy consequence of the gauged $U(1)_{R}$ may only be the presence of a discrete gauge symmetry such as $R$ parity. However, it is not completely clear whether the $U(1)_{R}$ symmetry is necessarily broken in the present theory, and if it remains unbroken at low energies we would have a massless $U(1)_{R}$ gauge boson which couples only to the superpartners and conjugate superpartners.

\section{Conclusions}

In this paper we have proposed an embedding of the standard model in a supersymmetric theory with an extra dimension compactified on the orbifold $S^{1} /\left(Z_{2} \times Z_{2}^{\prime}\right)$ - a circle with two orthogonal reflection symmetries. All standard model particles propagate in the bulk, yet, because the compactification breaks supersymmetry, they are the only massless modes at tree level. The only interactions in the bulk are the 5 d supersymmetric $S U(3) \times S U(2) \times U(1)$ gauge interactions, which lead at low energies to the standard model gauge interactions and the tree-level Higgs potential $\left(g^{2}+g^{\prime 2}\right)\left|\phi_{H}\right|^{4} / 8$. The orbifold allows for two different types of hypermultiplet, according to whether the zero mode is a scalar or a fermion. Thus the orbifold provides a distinction between the Higgs and lepton doublet superfields.

The Yukawa couplings for up-type quarks appear as 4d supersymmetric interactions at the fixed points of the $Z_{2}$ symmetry, while the Yukawa couplings for down-type quarks and charged leptons appear as $4 \mathrm{~d}$ supersymmetric interactions at the fixed points of the $Z_{2}^{\prime}$ symmetry. Because supersymmetry acts differently at these spatially separated locations, all Yukawa interactions involve just a single Higgs doublet, $\phi_{H}$. The top quark KK tower induces a one-loop effective potential for the Higgs which depends on the compactification scale. This potential contains a negative mass-squared, and is a monotonically decreasing function of $\left|\phi_{H}\right|$. It triggers EWSB, but runaway behaviour is prevented by the stabilizing effect of the tree-level gauge quartic interaction. Requiring the minimum to give the observed value for the Fermi coupling determines the compactification scale: $R^{-1}=352 \pm 20 \mathrm{GeV}$, leading to a prediction for the Higgs mass of $m_{H}=127 \pm 8 \mathrm{GeV}$. It is remarkable that the Higgs potential does not have the standard model form, and the dependence of the top quark mass on the Higgs VEV is also non-standard.

The nature of the orbifold automatically ensures that supersymmetry is broken and that the superpartners all have mass $R^{-1}$ at tree level. There is no additional supersymmetry breaking sector or mediation mechanism, and hence no soft supersymmetry breaking parameters such as $\mu, m_{1 / 2}, m_{0}, A$ or $B$. There is no fine tuning between parameters for successful EWSB, as there 
is only a single free parameter, $R^{-1}$. Because supersymmetry is broken non-locally by the global properties of the orbifold, all supersymmetry breaking effects are finite and reliably calculated in terms of physics at the compactification scale. Unlike $4 \mathrm{~d}$ supersymmetric theories, which have a large logarithmic dependence on physics at high energies, supersymmetry breaking effects are exponentially shielded from physics above $R^{-1}$.

There are three Dirac charginos of mass $(352,370,370) \mathrm{GeV}$ and three Dirac neutralinos of mass $(352,364,364) \mathrm{GeV}$, where the splitting is induced by EWSB. The EWSB splittings are largest for the top squarks, with two having a mass of $197 \pm 20 \mathrm{GeV}$ and two having a mass of $522 \mathrm{GeV}$. All other superpartners have a mass close to $R^{-1}$, while all $\mathrm{KK}$ excitations of the standard model have a mass close to $2 R^{-1}=704 \pm 40$. The LSP is a top squark and is most likely stable. It will be copiously produced at future runs of the Tevatron collider.

Sensitivity to physics at energies much above $R^{-1}$ enters only through supersymmetric interactions at the orbifold fixed points. This gives an additional $20 \%$ uncertainty to all the masses given above, except for the masses of the top squarks and the Higgs boson, which are more rigidly tied to EWSB. Higher dimension operators also contribute to the $\rho$ parameter, and must cancel contributions from the superpartners and KK excitations at the level of one order of magnitude. This UV sensitivity implies that $\rho$ cannot be reliably computed.

Our theory becomes non-perturbative at a scale of about $2 \mathrm{TeV}$, above which it becomes incorporated in some other higher dimensional theory. This might be a quasi-conformal field theory with an energy desert, or it could be that the fundamental scale of gravity is not far above $2 \mathrm{TeV}$ [12].

\section{Acknowledgements}

We would like to thank N. Arkani-Hamed, R. Rattazzi, D. Smith and N. Weiner for discussions. Y.N. thanks the Miller Institute for Basic Research in Science for financial support. This work was supported by the E.C. under the RTN contract HPRn-CT-2000-00148, the Department of Energy under contract DE-AC03-76SF00098 and the National Science Foundation under contract PHY-95-14797. 


\section{References}

[1] LEP Electroweak Working Group, http://lepewwg.web.cern.ch/LEPEWWG/.

[2] L. Maiani, in Proc. of Gif-sur-Yvette Summer School, (Paris, France, 1980), p. 3; E. Witten, Nucl. Phys. B188 (1981) 513; M. Veltman, Acta. Phys. Pol. 12 (1981) 437.

[3] L. Ibanez and G.G. Ross, Phys. Lett. B110 (1982) 215; L. Alvarez-Gaume, M. Claudson and M.B. Wise, Nucl. Phys. B207 (1982) 96; K. Inoue, A. Kakuto, H. Komatsu and S. Takeshita, Prog. Theor. Phys. 67 (1982) 1889; Prog. Theor. Phys. 68 (1982) 927, Erratum-ibid. 70 (1983) 330.

[4] S. Dimopoulos and H. Georgi, Nucl. Phys. B193 (1981) 150.

[5] N. Sakai, Z. Phys. C11 (1981) 153; S. Dimopoulos, S. Raby and F. Wilczek, Phys. Rev. D24 (1981) 1681; L.E. Ibanez and G.G. Ross, Phys. Lett. B105 (1981) 439.

[6] T. Yanagida, in Proc. of the Workshop on the Unified Theory and Baryon Number in the Universe, ed. O. Sawada and A. Sugamoto (KEK report 79-18, 1979), p. 95; M. Gell-Mann, P. Ramond and R. Slansky, in Supergravity, ed. P. van Nieuwenhuizen and D.Z. Freedman (North Holland, Amsterdam, 1979), p. 315.

[7] H. Goldberg, Phys. Rev. Lett. 50 (1983) 1419; J. Ellis, J. Hagelin, D.V. Nanopoulos and M. Srednicki, Phys. Lett. B127 (1983) 233.

[8] I. Antoniadis, Phys. Lett. B246 (1990) 377.

[9] L. Dixon, J.A. Harvey, C. Vafa and E. Witten, Nucl. Phys. B261 (1985) 678; Nucl. Phys. B274 (1986) 285.

[10] N. Arkani-Hamed, L. Hall, Y. Nomura, D. Smith and N. Weiner, in preparation.

[11] K.R. Dienes, E. Dudas and T. Gherghetta, Phys. Lett. B436 (1998) 55, hep-ph/9803466; Nucl. Phys. B537 (1999) 47, hep-ph/9806292.

[12] N. Arkani-Hamed, S. Dimopoulos and G. Dvali, Phys. Lett. B429 (1998) 263, hepph/9803315; Phys. Rev. D59 (1998) 086004, hep-ph/9807344; I. Antoniadis, N. ArkaniHamed, S. Dimopoulos and G. Dvali, Phys. Lett. B436 (1998) 257, hep-ph/9804398.

[13] I. Antoniadis, C. Munoz and M. Quiros, Nucl. Phys. B397 (1993) 515, hep-ph/9211309; K. Benakli, Phys. Lett. B386 (1996) 106, hep-th/9509115; I. Antoniadis, S. Dimopoulos and G. Dvali, Nucl. Phys. B516 (1998) 70, hep-ph/9710204.

[14] J. Scherk and J.H. Schwarz, Phys. Lett. B82 (1979) 60; Nucl. Phys. B153 (1979) 61.

[15] A. Pomarol and M. Quiros, Phys. Lett. B438 (1998) 255, hep-ph/9806263; A. Delgado, A. Pomarol and M. Quiros, Phys. Rev. D60 (1999) 095008, hep-ph/9812489. 
[16] I. Antoniadis, S. Dimopoulos, A. Pomarol and M. Quiros, Nucl. Phys. B544 (1999) 503, hep-ph/9810410.

[17] I. Antoniadis and K. Benakli, Phys. Lett. B326 (1994) 69, hep-th/9310151; I. Antoniadis, K. Benakli and M. Quiros, Phys. Lett. B331 (1994) 313, hep-ph/9403290; Phys. Lett. B460 (1999) 176, hep-ph/9905311; E. Accomando, I. Antoniadis and K. Benakli, Nucl. Phys. B579 (2000) 3, hep-ph/9912287.

[18] P. Nath and M. Yamaguchi, Phys. Rev. D60 (1999) 116004, hep-ph/9902323; Phys. Rev. D60 (1999) 116006, hep-ph/9903298; P. Nath, Y. Yamada and M. Yamaguchi, Phys. Lett. B466 (1999) 100, hep-ph/9905415.

[19] W.J. Marciano, hep-ph/9902332; Phys. Rev. D60 (1999) 093006, hep-ph/9903451; M. Masip and A. Pomarol, Phys. Rev. D60 (1999) 096005, hep-ph/9902467; T.G. Rizzo and J.D. Wells, Phys. Rev. D61 (2000) 016007, hep-ph/9906234; A. Strumia, Phys. Lett. B466 (1999) 107, hep-ph/9906266; R. Casalbuoni, S. De Curtis, D. Dominici and R. Gatto, Phys. Lett. B462 (1999) 48, hep-ph/9907355; C.D. Carone, Phys. Rev. D61 (2000) 015008, hep-ph/9907362.

[20] A. Delgado, A. Pomarol and M. Quiros, JHEP 0001 (2000) 030, hep-ph/9911252.

[21] P. Fayet, Phys. Lett. B159 (1985) 121; Nucl. Phys. B263 (1986) 649.

[22] Z. Chacko, M.A. Luty and E. Ponton, JHEP 0007 (2000) 036, hep-ph/9909248.

[23] W. Beenakker, M. Kramer, T. Plehn and M. Spira, hep-ph/9810290; M. Spira, hepph/9812407.

[24] R. Culbertson et al., hep-ph/0008070.

[25] A. Connolly, CDF Collaboration, hep-ex/9904010.

[26] L.J. Hall and L. Randall, Nucl. Phys. B352 (1991) 289; A.H. Chamseddine and H. Dreiner, Nucl. Phys. B458 (1996) 65, hep-ph/9504337; D.J. Castano, D.Z. Freedman and C. Manuel, Nucl. Phys. B461 (1996) 50, hep-ph/9507397.

[27] D.Z. Freedman, Phys. Rev. D15 (1977) 1173; P. Breitenlohner, Nucl. Phys. B124 (1977) 500; B. de Wit and P. van Nieuwenhuizen, Nucl. Phys. B139 (1978) 216.

[28] P. Breitenlohner and M.F. Sohnius, Nucl. Phys. B178 (1981) 151; B. de Wit, J.W. van Holten and A. Van Proeyen, Nucl. Phys. B184 (1981) 77, Erratum-ibid. B222 (1983) 516. 